\title{
Diffusion of spheres in isotropic and nematic networks of rods: Electrostatic interactions and hydrodynamic screening
}

\author{
Kyongok Kang \\ Forschungszentrum Jülich, Institute für Festkörper Forschung (IFF), Weiche Materie, \\ D-52425 Jülich, Germany \\ A. Wilk ${ }^{\text {a) }}$ and A. Patkowski \\ Institute of Physics, A. Mickiewicz. University, Umultowska 85, 61-614 Poznan, Poland \\ Jan K. G. Dhont \\ Forschungszentrum Jülich, Institute für Festkörper Forschung (IFF), Weiche Materie, \\ D-52425 Jülich, Germany
}

(Received 11 January 2007; accepted 12 April 2007; published online 1 June 2007)

\begin{abstract}
Translational diffusion of a small charged tracer sphere in isotropic and nematic suspensions of long and thin charged rods is investigated as a function of ionic strength and rod concentration. A theory for the diffusive properties of a small sphere is developed, where both (screened) hydrodynamic interactions and charge interactions between the tracer sphere and the rod network are analyzed. Hydrodynamic interactions are formulated in terms of the hydrodynamic screening length. As yet, there are no independent theoretical predictions for the hydrodynamic screening length for rod networks. Experimental tracer-diffusion data are presented for various ionic strengths as a function of the rod concentration, both in the isotropic and nematic states. Orientational order parameters are measured for the same ionic strengths as a function of the rod concentration. The hydrodynamic screening length is determined from these experimental data and scaling relations obtained from the above mentioned theory. For the isotropic networks, a master curve is found for the hydrodynamic screening length as a function of the rod concentration. For the nematic networks the screening length turns out to be a very sensitive function of the orientational order parameter. () 2007 American Institute of Physics. [DOI: 10.1063/1.2737446]
\end{abstract}

\section{INTRODUCTION}

The majority of reported tracer-diffusion experiments of spherical particles in rod networks focus on proteins in suspensions of F-actin, ${ }^{1-10}$ which are relevant for mass transport in cells. Recently, experiments on tracer diffusion of spheres in host suspensions of slender particles, other than F-actin, have been reported: nucleosome core particles in dispersions of DNA, ${ }^{11}$ colloidal spheres in solutions of "living polymers," 12 and colloidal spheres in dispersions of xanthan. ${ }^{13}$ "Histone tails" fold out of the nucleosome particles at a certain ionic strength and adsorb to DNA, which gives rise to anomalous stretched exponential diffusive behavior. ${ }^{11}$ The living polymers of Ref. 12 consist of selfassociating monomers bis(ethylhexylureido)toluene (EHUT). Both the polymerization/depolymerization kinetics and the length distribution of EHUT probably have a strong influence on the diffusive properties of the tracer spheres. The xanthan host dispersions in Ref. 13, where short-time translational and rotational diffusion coefficients of a tracer sphere are measured, can be regarded as a polymer network.

The host networks in the above-mentioned references exhibit a quite complicated dynamics by themselves. As far as we know, there are no experimental data available on

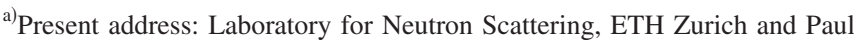
Scherrer Institute, CH-5232 Villigen, PSI, Switzerland.
}

tracer diffusion of colloidal spheres in the much more simple networks consisting of relatively stiff, very long, and thin rods, aside from two earlier papers of the present authors. ${ }^{14,15}$ In these two papers and in the present study we use fd virus as a rodlike colloidal host particle, which is indeed a very long and thin, rather stiff, rod and does not exhibit polymerization/depolymerization nor possible bundle formation (like F-actin, wormlike micelles and EHUT). Moreover, due to the very small van der Waals attractions between the fd-virus particles, the rod networks are stable over a wide range of salt concentrations, which offers the possibility to study diffusion in these networks as a function of the range of electrostatic interactions. The experiments in Refs. 14 and 15 were all done at relatively high salt concentrations, leading to essentially hard-core interactions between the tracer sphere and the rods.

Fd-virus suspensions have been recognized before as very good model systems for monodisperse rod dispersions in connection to (micro-) rhelogy ${ }^{16-19}$ and phase transitions (sometimes as binary mixtures with spherical colloids or polymers). ${ }^{20-23}$ Reviews of the phase behavior of fd-virus suspensions can be found in Refs. 24-26.

There are a few (semi-) phenomenological theories on translational diffusion of tracer spheres through networks. ${ }^{27-31}$ In Refs. 14 and 15 of the present authors, an attempt has been made to develop a microscopic theory for tracer diffusion of spheres through relatively simple net- 


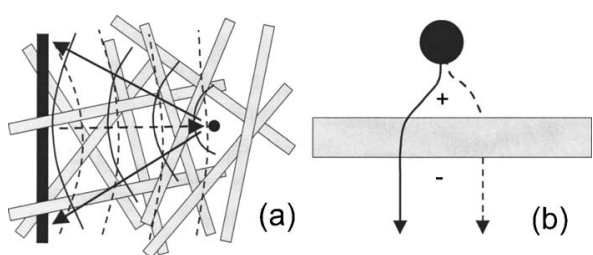

FIG. 1. (a) Hydrodynamic interactions between the tracer sphere and a rod (in black) in the network. The moving tracer sphere induces a flow field (solid lines) that is reflected by a rod back to the sphere (dashed lines). Hydrodynamic interaction is screened by the entangled rods in between the sphere and the (black) rod under consideration. (b) The "shadowing effect:" the probability to find a sphere that moves past a rod on the upper side of the rod is larger than on the shadow side.

works of stiff, long, and thin rods, where both (screened) hydrodynamic interactions and direct interactions between the tracer sphere and the rod network are explicitly accounted for.

There are fundamentally different mechanisms leading to the slowing down of diffusion of a tracer sphere due to the presence of a rod network, depending on whether the tracer sphere is large or small in comparison to the mesh size of the network. For large tracer spheres, the translational motion of the tracer sphere is only possible when the network structure is severely distorted. In this case, direct interactions between the sphere and the rods are much more pronounced than hydrodynamic interactions. Tracer diffusion can then be described with the neglect of hydrodynamic interactions. ${ }^{14}$ For small tracer spheres, where the sphere can move through the voids of the network without distorting the structure of the network, hydrodynamic interactions are important [see Fig. 1(a)]. Due to the entanglement of the rods in the network, hydrodynamic interactions between the tracer sphere and the rods are screened. This screening is characterized by the socalled hydrodynamic screening length, which measures the penetration depth of shear waves into the network. For the rod networks considered here, there is no theory available yet for the hydrodynamic screening length. Despite the fact that the network structure remains essentially unchanged during the diffusive motion of such a small sphere, there is nevertheless a distortion of the pair-correlation function between the sphere and the rods. This distortion is due to the fact that when the sphere moves past a rod, the probability to find the sphere on the side of approach of the rod is larger than that on the "shadow side" of the rod, as depicted in Fig. 1(b). The rod-sphere pair-correlation function is therefore distorted, giving rise to a force on the sphere that affects its diffusive motion. We referred to the distortion of the pair-correlation function in Ref. 15 as "the shadowing effect." The shadowing contribution is determined not only by hard-core interactions, but also by charge interactions in case the rods and the sphere carry surface charges. The shadowing effect can thus be varied systematically by changing the electrostatic screening length through variation of the ionic strength of the rod suspensions.

In the present paper we investigate long-time translational tracer diffusion of a charged tracer sphere in networks of charged rods at various ionic strengths. The theory on the effect of hydrodynamic interactions, as well as direct inter- actions, including charge interactions, is described in Sec. II. This is an extension of the theory developed in Ref. 15 to include charge-interaction contributions to the diffusion coefficient. The rather complicated expressions for the diffusion coefficient are simplified in Sec. III where scaling relations are derived. The use of these scaling relations greatly reduces the efforts involved in the data analysis. A summary of the relevant scaling relations which are used in our analysis of experimental data is given in Sec. IV. Section V is the experimental section. The colloidal systems are shortly described in Sec. V A. Carbon dioxide that dissolves from the air contributes significantly to the ionic strength and the $p \mathrm{H}$ at low ionic strengths which, in turn, affects the surface charges of the rods (fd-virus particles) we used. This is discussed in Sec. V B. The orientational order parameter plays an important role for tracer diffusion in nematic networks. The shadowing contribution depends explicitly on the order parameter, while the hydrodynamic contribution depends implicitly on the order parameter through the hydrodynamic screening length. The orientational order parameter as a function of rod concentration and ionic strength is presented in Sec. V C. The measured orientational order parameters are compared to data known from literature at relatively high ionic strengths. Section V D presents the data on long-time self-diffusion for various ionic strengths as functions of the rod concentration both in the isotropic and nematic phase. Section VI contains the data analysis, where the hydrodynamic screening length is calculated from our experimental diffusion coefficients. A summary and conclusions are given in Sec. VII.

\section{THEORY}

In the long-time limit, on time scales much larger than the microstructural relaxation times of the rod network, the self-diffusion coefficient $D_{s}$ is equal to

$$
D_{s}=\frac{k_{B} T}{\gamma_{p}},
$$

where $k_{B}$ is Boltzmann's constant, $T$ is the temperature, and $\gamma_{p}$ is the friction coefficient of the tracer particle with the solvent and the rod network. This friction coefficient is the proportionality constant between the external force $\mathbf{F}^{\text {ext }}$ acting on the tracer sphere and its resulting thermally averaged velocity $\left\langle\mathbf{v}_{p}\right\rangle$,

$$
\mathbf{F}^{\mathrm{ext}}=\gamma_{p}\left\langle\mathbf{v}_{p}\right\rangle \text {. }
$$

The friction coefficient is affected by hydrodynamic interactions and direct interactions of the tracer particle with the rod network.

The effect of screened hydrodynamic interactions is described in Ref. 15 and will be summarized in the following section. It will be shown that electrostatic interactions have negligible influence on the hydrodynamic-interaction contribution to the self-diffusion coefficient once the rod concentration and the order parameter (in the case of a nematic network) are given. The shadowing effect, however, is strongly altered due to electrostatic interactions, which is considered in Sec. II B. 


\section{A. Hydrodynamic interactions}

When the tracer sphere moves through the solvent, it creates a fluid flow that will be reflected back to the sphere by the rods in the network. The sphere experiences an additional friction due to this reflected flow field. This additional friction determines the effect of hydrodynamic interactions on the diffusion coefficient. Due to the long-ranged nature of hydrodynamic interactions, the major contribution from reflected flow originates from rods that are many mesh sizes away from the tracer sphere. Both the flow from the sphere to a rod and that back to the sphere thus typically experience the presence of many intermediate rods [see Fig. 1(a)]. As a result of entanglement of these rods, hydrodynamic interaction between the tracer sphere and a given rod in the network is screened by the rods that are in between the sphere and the given rod [in black in Fig. 1(a)]. If the hydrodynamic screening length is larger than the mesh size of the network, the intermediate network can be described as "an effective" medium on which a body force acts proportionally to the local fluid flow velocity. As shown in Ref. 15, for a specified velocity of the tracer particle equal to $\left\langle\mathbf{v}_{p}\right\rangle$, the external force required to maintain this velocity is equal to

$$
\mathbf{F}^{\mathrm{ext}}=\gamma_{0}\left[\hat{\mathbf{I}}+\sum_{j=1}^{N}\left\langle\mathbf{M}\left(\mathbf{R}_{j}, \hat{\mathbf{u}}_{j}\right)\right\rangle\right] \cdot\left\langle\mathbf{v}_{p}\right\rangle,
$$

where $\gamma_{0}=6 \pi \eta_{0} a$ is the friction coefficient of the tracer with the fluid in the absence of the rods (with $\eta_{0}$ the shear viscosity of the fluid and $a$ the radius of the tracer sphere) and $\hat{\mathbf{I}}$ is the identity tensor. Furthermore, the summation is over all rods in the network, and the tensor $\mathbf{M}$ accounts for screened hydrodynamic interactions between the sphere and a rod,

$$
\begin{aligned}
\mathbf{M}(\mathbf{R}, \hat{\mathbf{u}})= & \frac{12\left(\pi \eta_{0}\right)^{2} a}{G(\kappa D, \kappa L)} \int_{-(1 / 2) L}^{(1 / 2) L} d l \mathbf{T}_{s}(\mathbf{R}-l \hat{\mathbf{u}}) \\
& \cdot\left\{\hat{\mathbf{I}}-\frac{H(\kappa D, \kappa L)}{G(\kappa D, \kappa L)+H(\kappa D, \kappa L)} \hat{\mathbf{u}} \hat{\mathbf{u}}\right\} \\
& \cdot \mathbf{T}_{s}(\mathbf{R}-l \hat{\mathbf{u}}),
\end{aligned}
$$

where $D$ and $L$ are the thickness and length of the rods, respectively, and $\mathbf{T}_{s}$ is the screened Oseen tensor,

$$
\begin{aligned}
& \mathbf{T}_{s}(\mathbf{r})=\frac{1}{4 \pi \eta_{0} r}\left[h_{1}(x) \hat{\mathbf{I}}+h_{2}(x) \hat{\mathbf{r}} \hat{\mathbf{r}}\right], \\
& h_{1}(x)=-x^{-2}+\exp \{-x\}\left(1+x^{-1}+x^{-2}\right), \\
& h_{2}(x)=3 x^{-2}-\exp \{-x\}\left(1+3 x^{-1}+3 x^{-2}\right),
\end{aligned}
$$

where $x=\kappa r$, with $\kappa^{-1}$ as the hydrodynamic screening length. Here, the subscript $s$ stands for "screening." Furthermore, the functions $G$ and $H$ are equal to

$$
G(\kappa D, \kappa L)=\int_{(1 / 2) \kappa D}^{(1 / 2) \kappa L} d x \frac{h_{1}(x)}{x},
$$

$$
H(\kappa D, \kappa L)=\int_{(1 / 2) \kappa D}^{(1 / 2) \kappa L} d x \frac{h_{2}(x)}{x} .
$$

The hydrodynamic screening length is a measure for the penetration depth of flow into the rod network. So far, there is no independent theory which predicts the rod-concentration and orientational-order-parameter dependence of the hydrodynamic screening length $\kappa^{-1}$. For short distances or large hydrodynamic screening lengths,

$$
h_{1}(x)=1 / 2=h_{2}(x) \quad \text { for } x \downarrow 0,
$$

in which case the screened Oseen tensor [Eq. (5)] reduces to the Oseen tensor for the creeping flow equation in the absence of body forces due to entanglement.

The tensor $\mathbf{M}$ is proportional to the radius $a$ of the tracer sphere because the additional friction force due to the reflected flow field is proportional to the size of the sphere.

The assumption made in the derivation of the above results is that flow fields that are reflected by a rod to another rod back to the sphere are relatively weak and are neglected. For the open network under consideration here this is probably a good approximation.

For a nematic network of rods the screening length is, in principle, anisotropic. However, since the flow induced by a sphere is stronger in the direction of motion of the sphere, it is assumed here that the most important contributions to hydrodynamic interactions relate to the fluid flow in the direction of motion of the tracer sphere, which is either parallel or perpendicular to the director of the nematic network. The validity of this assumption needs further theoretical work, where the Green's function of the Debye-Büche-Brinkman equation with an anisotropic screening length should be computed. As far as we know, this has not been done yet.

Since for the small tracer sphere considered here, for which the network structure is essentially unaffected by the presence of the sphere, the ensemble average in Eq. (3) can be taken with respect to the probability density function (pdf) of the degrees of freedom of a rod and the sphere that is not affected by the external force that acts on the tracer sphere. This leads to the following expression for the long-time selfdiffusion coefficient $D_{s}^{h}$, where only hydrodynamic interactions are accounted for (as indicated by the superscript $h$ ):

$$
\frac{D_{s}^{h}}{D_{0}}=\frac{1}{1+\alpha^{h} \varphi},
$$

where $\varphi=(\pi / 4) D^{2} L \bar{\rho}$ is the volume fraction of rods $(\bar{\rho}$ the number density of rods) and the coefficient $\alpha^{h}$ is given by

$$
\begin{aligned}
\alpha^{h}= & \frac{4}{\pi D^{2} L} \hat{\mathbf{v}}_{p} \cdot\left[\int d \mathbf{R} \oint d \hat{\mathbf{u}} P_{0}(\hat{\mathbf{u}} \mid S)\right. \\
& \times \exp \{-\beta V(\mathbf{R}, \hat{\mathbf{u}})\} \mathbf{M}(\mathbf{R}, \hat{\mathbf{u}})] \cdot \hat{\mathbf{v}}_{p},
\end{aligned}
$$

where $\hat{\mathbf{v}}_{p}=\left\langle\mathbf{v}_{p}\right\rangle /\left|\left\langle\mathbf{v}_{p}\right\rangle\right|$ is the unit vector along the ensemble averaged velocity of the tracer particle, $P_{0}(\hat{\mathbf{u}} \mid S)$ is the pdf for the orientation $\hat{\mathbf{u}}$ of a rod in the undistorted network (as indicated by the subscript " 0 "), $\beta=1 / k_{B} T$ (with $k_{B}$ Boltzmann's constant and $T$ the temperature), and $V\left(\mathbf{R}=\mathbf{r}_{p}\right.$ 
$\left.-\mathbf{r}_{c}, \hat{\mathbf{u}}\right)$ is the pair-interaction potential for a rod with position coordinate $\mathbf{r}_{c}$ and the tracer sphere with position $\mathbf{r}_{p}$.

Note that compared to Ref. 15, the pdf $P_{0}(\hat{\mathbf{u}} \mid S)$ for the orientation $\hat{\mathbf{u}}$ of a rod is denoted here as a function of the scalar orientation order parameter $S$ rather than the rod concentration.

In the above results it is assumed that the force on the tracer sphere is colinear with its average velocity. This is the case we consider in the experimental section, where diffusion in an isotropic network is considered, as well as diffusion in a nematic rod suspension either parallel or perpendicular to the nematic director.

In Sec. III A we shall reduce the rather complicated form in Eq. (9) to a much simpler form by scaling arguments.

\section{B. The shadowing contribution}

As a sphere passes along a rod, the probability to find a tracer sphere on the side of the rod from which the sphere approaches the rod is higher than on the other shadow side of the rod, as depicted in Fig. 1(b). This difference in probability, as quantified by the pair-correlation function, induces a force on the spheres that affects the diffusion coefficient, which is referred to here as the shadowing effect. As discussed in Ref. 15, there are two different configurations of the rod relative to the external force acting on the tracer sphere that need to be considered: $\hat{\mathbf{u}} \| \mathbf{F}^{\text {ext }}$ and $\hat{\mathbf{u}} \perp \mathbf{F}^{\mathrm{ext}}$. In the case where $\hat{\mathbf{u}} \| \mathbf{F}^{\mathrm{ext}}$ the distortion of the pair-correlation function is very small as compared to the case where $\hat{\mathbf{u}} \perp \mathbf{F}^{\text {ext }}$, provided the rods are very long and thin. We shall therefore only consider the case where $\hat{\mathbf{u}} \perp \mathbf{F}^{\text {ext }}$. The distortion of the pair-correlation function for arbitrary orientations of the rod will then be constructed from this special case. For the very long and thin rods and the small diameter of the tracer sphere as compared to the length of the rods, end effects can be neglected. The rod is thus taken infinitely long in the analysis given below.

In the overdamped limit, where inertial forces can be neglected, the total force on the tracer sphere is zero. That is, the external force $\mathbf{F}^{\text {ext }}$, the force $-\nabla V$ due to interaction with a rod (with $\nabla$ the gradient operator with respect to the position coordinate of the tracer sphere), the Brownian force $-k_{B} T \nabla \ln g$ (with $g$ as the pair-correlation function), and the hydrodynamic force $\mathbf{F}^{h}$ add up to zero,

$$
\mathbf{F}^{\mathrm{ext}}-\nabla V-k_{B} T \nabla \ln g+\mathbf{F}^{h}=0 .
$$

The hydrodynamic force has been calculated in the previous section as

$$
\mathbf{F}^{h}=-\gamma^{h} \mathbf{v}_{p}, \quad \gamma^{h}=1+\alpha^{h} \varphi,
$$

where $\mathbf{v}_{p}$ is the translational velocity of the sphere and the coefficient $\alpha^{h}$ is given in Eq. (9). Using this expression for the hydrodynamic force amounts to the assumption that the tracer particle can be regarded as moving through an effective fluid with a friction coefficient equal to $\gamma^{h}$, which accounts for friction with the solvent, as well as hydrodynamic interactions with the rod network. From the force balance equation [Eq. (10)] it thus follows that $\mathbf{v}_{p}=D_{s}^{h}\left[\beta \mathbf{F}^{\text {ext }}-\beta \nabla V\right.$ $-\nabla \ln g]$, where, as before, $D_{s}^{h}=k_{B} T / \gamma^{h}$ is the diffusion co-

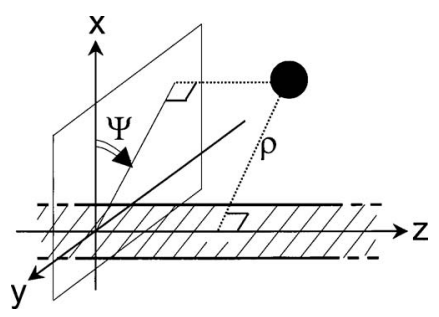

FIG. 2. Definition of the cylindrical coordinates for the pair-correlation function: $\rho$ is the shortest distance between the center line of the rod and the center of the sphere and $\Psi$ is the angle between the projection of the position vector of the sphere onto the $x y$ plane and the positive $x$ axis. The external force acting on the tracer sphere is directed along the negative $x$ direction.

efficient where only hydrodynamics is accounted for. As it turns out, the self-diffusion coefficient $D_{s}$, where both hydrodynamic interactions and shadowing are accounted for, can be written as

$$
\frac{D_{s}}{D_{0}}=\frac{D_{s}^{h}}{D_{0}} \frac{D_{s}^{s}}{D_{0}},
$$

where $D_{s}^{s}$ is the diffusion coefficient related to the shadowing effect (the superscript $s$ stands for shadowing). The diffusion coefficient where both hydrodynamic and direct interactions are accounted for follows from

$$
\left\langle\mathbf{v}_{p}\right\rangle=D_{s}^{h}\left[\beta \mathbf{F}^{\text {ext }}-\beta\langle\nabla V\rangle-\langle\nabla \ln g\rangle\right]
$$

When the distortion of the pair correlation is neglected, $g$ is equal to $g=\exp \{-\beta V\}$ so that Eq. (13) reduces to $\left\langle\mathbf{v}_{p}\right\rangle$ $=D_{s}^{h} \beta \mathbf{F}^{\text {ext }}$, which implies that the diffusion coefficient is equal to $D_{s}^{h}$, as it should. Including the shadowing effect accounts for the additional contribution to friction as a result of direct interactions of the tracer sphere with the rods.

The ensemble average in Eq. (13) can be evaluated once $g$ is known explicitly. The pair-correlation function can be obtained from the continuity equation

$$
\frac{\partial g}{\partial t}=\nabla \cdot\left(\mathbf{v}_{p} g\right)=D_{s}^{h} \nabla \cdot\left[\beta g \mathbf{F}^{\mathrm{ext}}-\beta g \nabla V-\nabla g\right]
$$

Neglecting end effects, the pair-correlation function is a function of the cylindrical coordinates $\Psi$ and $\rho$, as defined in Fig. 2. Since we are interested in the linear relationship between the external force on the tracer sphere and the resulting velocity, equations can be linearized with respect to $\mathbf{F}^{\mathrm{ext}}$. The pair-correlation function is therefore written as [with $g_{0}(\rho)=\exp \{-\beta V(\rho)\}$ as the undistorted pair-correlation function]

$$
g(\rho, \Psi)=g_{0}(\rho)\left[1+\cos \{\Psi\} L(\rho) F^{\mathrm{ext}}\right] .
$$

Substitution into Eq. (14) and transforming to cylindrical coordinates as defined in Fig. 2 (using $\beta g \nabla V+\nabla g$ $=g_{0} \nabla\left[\cos \{\Psi\} L(\rho) F^{\text {ext }}\right]$ which follows from $\left.\nabla g_{0}=-\beta g_{0} \nabla V\right)$ leads to the following equation for the unknown function $L(\rho)$ : 


$$
\begin{gathered}
g_{0}(\rho)\left[\frac{d L^{2}(\rho)}{d \rho^{2}}+\frac{1}{\rho} \frac{d L(\rho)}{d \rho}-\frac{L(\rho)}{\rho^{2}}\right] \\
=-\frac{d g_{0}(\rho)}{d \rho}\left[\beta+\frac{d L(\rho)}{d \rho}\right] .
\end{gathered}
$$

This equation should be solved for $L(\rho)$ in order to calculate the ensemble averages in Eq. (13).

For hard-core interactions, in the absence of charges, $g_{0}(\rho)=1$ for $\rho>a+D / 2$, so that the right-hand side of Eq. (16) is 0 for these distances (with $a$ as the radius of the tracer sphere and $D$ as the diameter of the rod). This allows the integration of Eq. (16), where the integration constants are determined from the boundary condition $L(\rho) \rightarrow 0$ as $\rho \rightarrow \infty$ and from an integration of Eq. (16) from $a+D / 2-\epsilon$ to $a$ $+D / 2+\epsilon$ for vanishing $\epsilon$. The solution for hard-core potentials is then found to be $\mathrm{e}^{15}$ (the index "hc" stands for hard core)

$$
L_{\mathrm{hc}}(\rho)=\frac{\beta[a+D / 2]^{2}}{\rho} .
$$

The middle term on the left-hand side in Eq. (16) is erroneously missing in similar Eqs. (39) and (40) in Ref. 15. All equations in Ref. 15 remain valid, however, when $\nu$ is set equal to 1 . Since the shadowing effect for the case considered in Ref. 15 is very small, this does not alter any of its conclusions.

In the present case, in addition to hard-core interactions, interactions due to charges on the surfaces of the tracer sphere and the rod must be accounted for. For sufficiently small surface charges the interaction potential $V_{Q}$ between the tracer sphere and the rod is a superposition of DebyeHückel interaction potentials,

$$
\beta V_{Q}(\rho)=K \int_{-\infty}^{\infty} d x \frac{\exp \left\{-\kappa_{Q} \sqrt{\rho^{2}+x^{2} D^{2}}\right\}}{\sqrt{\rho^{2}+x^{2} D^{2}}},
$$

where the constant $K$ is equal to

$$
K=Z_{c} Z_{\mathrm{rod}} \frac{D}{L} l_{B} \frac{\exp \left\{\kappa_{Q}(a+D / 2)\right\}}{\left(1+\kappa_{Q} a\right)\left(1+\kappa_{Q} D / 2\right)},
$$

where $l_{B}=\beta e^{2} / 4 \pi \epsilon$ is the Bjerrum length $(0.71 \mathrm{~nm}$ for water at room temperature), with $\epsilon$ as the static dielectric constant of the solvent, $e>0$ the elementary charge, and $Z_{c}$ and $Z_{\mathrm{rod}}$ the number of elementary charges on the surface of the tracer sphere and a rod, respectively (including the sign of the charge). Furthermore, $\kappa_{Q}$ is the inverse Debye-Hückel electrostatic screening length, which is a function of number concentrations $\rho_{\alpha}^{0}$ of ion species $\alpha$ that carry $z_{\alpha}$ elementary charges $|e|$,

$$
\kappa_{Q}=\sqrt{\frac{e^{2}}{k_{B} T \epsilon} \sum_{\alpha} \rho_{\alpha}^{0} z_{\alpha}^{2}} .
$$

The index $Q$ on $\kappa$ is used here to distinguish it from the hydrodynamic screening length $\kappa^{-1}$. The bulk number concentrations $\rho_{\alpha}^{0}$ are the concentrations of ions in a reservoir that is in osmotic equilibrium with the suspension of rods. In the experiments, the fd-virus suspensions are always dialyzed for at least two days against an electrolyte solution with known ion concentrations $\rho_{\alpha}^{0}$ from which the electrostatic screening length is calculated.

The above expressions for the interaction potential are good approximations when typical potential energies of interaction $V_{Q}$ are at most a few times $k_{B} T$. The differential equation [Eq. (16)] will be solved to leading order in chargeinteraction energies, in line with the Debye-Hückel approximation involved in Eqs. (18) and (19). To this end $L(\rho)$ is written as

$$
L(\rho)=L_{\mathrm{hc}}(\rho)+L_{Q}(\rho),
$$

where $L_{\mathrm{hc}}(\rho)$ is the hard-core contribution in Eq. (17) and $L_{Q}(\rho)$ is the contribution due to charge interactions. Products of $L_{Q}$ and $V_{Q}$ in Eq. (16) are neglected. Appendix A contains the mathematical details in obtaining the following "DebyeHückel solution" of Eq. (16):

$$
L_{Q}(\rho)=-L_{\mathrm{hc}}(\rho)\left[F^{c}+\beta \int_{a+D / 2}^{\rho} d \rho^{\prime} L_{\mathrm{hc}}^{-1}\left(\rho^{\prime}\right) F\left(\rho^{\prime}\right)\right],
$$

where

$$
F\left(\rho^{\prime}\right)=\int_{\rho^{\prime}}^{\infty} d \rho^{\prime \prime} \frac{d V_{Q}\left(\rho^{\prime \prime}\right)}{d \rho^{\prime \prime}}\left[\beta-\frac{L_{\mathrm{hc}}\left(\rho^{\prime \prime}\right)}{\rho^{\prime \prime}}\right]
$$

and $F^{c}=F\left(\rho^{\prime}=a+D / 2\right)$ is "the contact value" of $F\left(\rho^{\prime}\right)$.

The ensemble averages in Eq. (13) can now be calculated in terms of charge-interaction parameters. As shown in Appendix B, to leading order in the charge-interaction parameters it is found that

$$
\begin{aligned}
\beta\langle\nabla V\rangle= & \varphi \beta F^{\operatorname{ext}}\left(1+\frac{2 a}{D}\right)^{2} \\
& \times\left\{1-\int_{a+D / 2}^{\infty} d \rho \frac{d V_{Q}(\rho)}{d \rho}\left[\beta-\frac{L_{\mathrm{hc}}(\rho)}{\rho}\right]\right\},
\end{aligned}
$$

$\langle\nabla \ln g\rangle=0$,

with $\varphi=(\pi / 4) D^{2} L \bar{\rho}$ as the volume fraction of rods.

The above calculation complies to a rod with a fixed orientation perpendicular to the direction of the external force. In the case of long and thin rods, the shadowing forces are much weaker for the motion of the sphere along the long axis of the rod and can be neglected. For arbitrary orientations of a rod, only the component of the external force perpendicular to the long axis of the rod needs to be considered. This component of the external force is equal to $[\hat{\mathbf{I}}$ - $\hat{\mathbf{u}} \hat{\mathbf{u}}] \cdot \mathbf{F}^{\text {ext }}$ and replaces $\mathbf{F}^{\text {ext }}$ in Eq. (24). Ensemble averaging with respect to the orientation of the rod gives

$$
\langle[\hat{\mathbf{I}}-\hat{\mathbf{u}} \hat{\mathbf{u}}]\rangle \cdot \mathbf{F}^{\mathrm{ext}}=\frac{2}{3}[\hat{\mathbf{I}}-\mathbf{Q}] \cdot \mathbf{F}^{\mathrm{ext}},
$$

where $\hat{\mathbf{I}}$ is the identity tensor and $\mathbf{Q}$ is the traceless orientational-order-parameter tensor, which is defined as

$$
\mathbf{Q} \equiv \frac{3}{2}\left\langle\hat{\mathbf{u}} \hat{\mathbf{u}}-\frac{1}{3} \hat{\mathbf{I}}\right\rangle \equiv \frac{3}{2} \oint d \hat{\mathbf{u}} P_{0}(\hat{\mathbf{u}} \mid S)\left[\hat{\mathbf{u}} \hat{\mathbf{u}}-\frac{1}{3} \hat{\mathbf{I}}\right],
$$

where, as before, $P_{0}(\hat{\mathbf{u}} \mid S)$ is the probability density function for the orientation of a rod, which depends on the order parameter $S$ (also commonly denoted as $P_{2}$ ). The orienta- 
tional order parameter is the largest eigenvalue of $\mathbf{Q}$. The ensemble average of $\nabla V$ in Eq. (24) thus generalizes for external forces with arbitrary orientation to

$$
\begin{aligned}
\beta\langle\nabla V\rangle= & \varphi \frac{2}{3}[\hat{\mathbf{I}}-\mathbf{Q}] \cdot \beta \mathbf{F}^{\mathrm{ext}}\left(1+\frac{2 a}{D}\right)^{2} \\
& \times\left\{1-\int_{a+D / 2}^{\infty} d \rho \frac{d V_{Q}(\rho)}{d \rho}\left[\beta-\frac{L_{\mathrm{hc}}(\rho)}{\rho}\right]\right\} .
\end{aligned}
$$

For an isotropic suspension of rods, $P_{0}(\hat{\mathbf{u}})=1 / 4 \pi$, so that $\mathbf{Q}=0$. For diffusion parallel to the nematic director, where $\mathbf{F}^{\text {ext }}$ is parallel to the director, $\mathbf{Q} \cdot \mathbf{F}^{\text {ext }}=S \mathbf{F}^{\text {ext }}$, where $S$ is the scalar orientational order parameter (sometimes also denoted as $P_{2}$ ). For diffusion perpendicular to the director, $\mathbf{Q} \cdot \mathbf{F}^{\mathrm{ext}}=$ $-\frac{1}{2} S \mathbf{F}^{\text {ext }}$.

From Eq. (13) and Eqs. (1) and (2) it follows that the self-diffusion coefficient, as far as the shadowing contribution is concerned, can indeed be written in the form of Eq. (12), with

$$
\frac{D_{s}^{s}}{D_{0}}=1-\alpha_{\text {iso }, \|, \perp}^{s} \varphi
$$

where

$$
\begin{aligned}
& \alpha_{\text {iso }}^{s}=R, \\
& \alpha_{\|}^{s}=R(1-S), \\
& \alpha_{\perp}^{s}=R\left(1+\frac{1}{2} S\right),
\end{aligned}
$$

for diffusion in an isotropic network and a nematic network parallel and perpendicular to the director, respectively. Here, the function $R$ follows from the explicit expressions derived above for the ensemble averages,

$$
R \equiv \frac{2}{3}\left(1+\frac{2 a}{D}\right)^{2}\left\{1-\int_{a+D / 2}^{\infty} d \rho \frac{d V_{Q}(\rho)}{d \rho}\left[\beta-\frac{L_{\mathrm{hc}}(\rho)}{\rho}\right]\right\},
$$

which is a function of the radius of the tracer sphere, the diameter of the rods, and the charge-interaction parameters; that is, the charges on the tracer sphere and rods and the Debye screening length. Since direct interactions with just a single rod are independently added, this result is the first term in an expansion with respect to the volume fraction. For long and thin rods, the volume fraction of interest is typically equal to $D / L$, which is indeed a small number.

\section{SCALING RELATIONS}

The hydrodynamic and shadowing contributions to the diffusion coefficient as they stand in Eqs. (8), (9), and (28) (30), respectively, are complicated expressions which require quite some numerical efforts to evaluate explicitly. In this section we shall derive approximations for these complicated expressions by scaling to dimensionless groups and analyze the dependence of resulting expressions on these groups.

\section{A. Scaling of the hydrodynamic contribution}

The mean-field concept of hydrodynamic screening is only meaningful when the hydrodynamic screening length $\kappa^{-1}$ is larger than the mesh size of the network. In that case, the tracer sphere interacts hydrodynamically with many different rods which are typically not in the immediate vicinity of the sphere. This implies that hydrodynamic forces are essentially unaffected by direct interactions between the tracer sphere and the rods. Mathematically, this can be seen from Eq. (9) for $\alpha^{h}$ as follows. For infinite hydrodynamic screening lengths, the integral in Eq. (9) diverges since in this limit the Oseen tensor $\mathbf{T}_{s}(\mathbf{R})$ varies as $\sim 1 / R$. Hence, for hydrodynamic screening lengths that are much larger than the range of the pair-interaction potential, the numerical value for $\alpha^{h}$ is essentially determined by contributions from large distances, larger than the electrostatic screening length. The numerical value of $\alpha^{h}$ is thus essentially independent of the details of the pair-interaction potential.

Since the mesh size of the network is larger than the diameter $D$ of the rods, the above described mean-field concept is only valid when $\kappa D$ is a very small number. In that case, the functions $G$ and $H$ in Eq. (6) reduce to $\frac{1}{2} \ln \{\kappa D\}$, provided $\kappa L$ is not a very large number (we will discuss this further in Appendix C). In that case the combination $H /(H$ $+G$ ) in Eq. (4) for $\mathbf{M}$ is equal to $1 / 2$, and the function $G$ in the prefactor to the integral renders a trivial prefactor $\sim \ln \{\kappa D\}$.

Transforming the integration variables $l$ and $\mathbf{R}$ in Eqs. (4) and (9) to the dimensionless variables $\kappa l$ and $\kappa \mathbf{R}$, respectively, and scaling the $1 / r$ dependence in Eq. (5) of the Oseen tensor to $1 / \kappa r$ then finally leads to a scaling relation of the form

$$
\alpha_{\mathrm{iso}, \|, \perp}^{h}=-\frac{\kappa a}{(\kappa D)^{2} \ln \{\kappa D\}} \Lambda_{\mathrm{iso}, \|, \perp}^{h}(\kappa L) .
$$

The scaling function $\Lambda^{h}$ is a well-behaved function of $\kappa D$ and is essentially independent of this dimensionless group because $\kappa D$ is a very small number (see Appendix B for further details). Furthermore, $\Lambda^{h}$ is essentially independent of the details of the pair-interaction potential as discussed above. Moreover, it turns out that $\Lambda^{h}$ is a weak function of the order parameter $S$ that enters explicitly through the orientational pdf in Eq. (9) (this is also discussed in detail in Appendix C). Of course, there remains an orientationalorder-parameter dependence implicitly through the hydrodynamic screening length.

From a numerical evaluation of $\alpha^{h}$, the scaling functions are calculated and plotted in Fig. 3(a). The following representations for the scaling functions are found (with $X=\kappa L$ ):

$$
\begin{aligned}
& \Lambda_{\text {iso }}^{h}(X)=\frac{64}{10}-\frac{85}{10000} X-\frac{33}{10000} X^{2}, \\
& \Lambda_{\|}^{h}(X)=\frac{53}{10}-\frac{95}{1000} X+\frac{3}{10000} X^{2},
\end{aligned}
$$



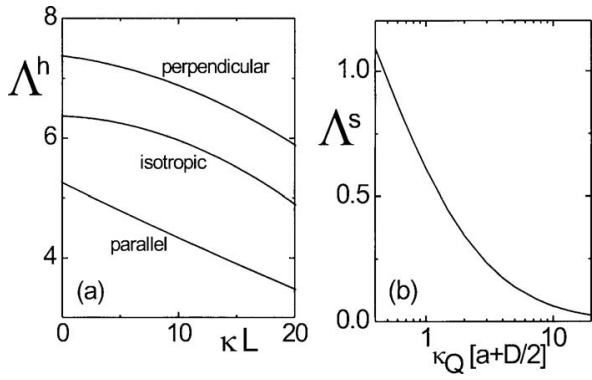

FIG. 3. (a) The hydrodynamic scaling functions vs $\kappa L$ and (b) the shadowing scaling function vs $X=\kappa_{Q}(a+D / 2)$. Note that the order-parameter dependence of the shadowing effect in the nematic phase enters explicitly in Eq. (43).

$$
\Lambda_{\perp}^{h}(X)=\frac{74}{10}-\frac{25}{1000} X-\frac{25}{10000} X^{2} .
$$

This representation is verified to be accurate to within about $5 \%$ by a numerical evaluation of $\alpha^{h}$ from Eq. (9) in the range $1 \leqslant \kappa L \leqslant 20$.

The above described approximations greatly simplify the analysis of experimental data and decouple hydrodynamic interactions from direct interactions.

\section{B. Scaling of the shadowing contribution}

Introducing the dimensionless distance $\tilde{\rho}=\rho /(a+D / 2)$ in Eq. (30), substitution of Eqs. (18) and (19) for the interaction potential and a partial integration leads to

$$
\begin{aligned}
R= & \frac{2}{3}\left(1+\frac{2 a}{D}\right)^{2} \\
& \times\left[1+2 \frac{l_{B}}{L} \frac{Z_{c} Z_{\mathrm{rod}}}{\left[1+\kappa_{Q} a\right]\left[1+\kappa_{Q} D / 2\right]} \Lambda^{s}\left(\kappa_{Q}[a+D / 2]\right)\right],
\end{aligned}
$$

where the scaling function $\Lambda^{s}$ is given by (the superscript $s$ stands for shadowing)

$$
\Lambda^{s}\left(\kappa_{Q}[a+D / 2]\right)=\exp \left\{\kappa_{Q}[a+D / 2]\right\} \int_{1}^{\infty} d \widetilde{\rho} W_{Q}(\widetilde{\rho}) / \widetilde{\rho}^{3},
$$

where $W_{Q}$ is "a dimensionless interaction potential" equal to

$$
W_{Q}(\widetilde{\rho})=\int_{-\infty}^{\infty} d \widetilde{x} \frac{\exp \left\{-\kappa_{Q}[a+D / 2] \sqrt{\tilde{\rho}^{2}+\tilde{x}^{2}}\right\}}{\sqrt{\tilde{\rho}^{2}+\widetilde{x}^{2}}},
$$

with $\tilde{x}=x D /(a+D / 2)$. As can be seen from these equations, the shadowing parameter $R$ depends on the dimensionless groups $Z_{c}, Z_{\text {rod }}, l_{B} / L, \kappa_{Q} a$, and $\kappa_{Q} D$. The dependence on the first three dimensionless groups is trivial, just being present in the prefactor to $\Lambda_{Q}$ in Eq. (35). The nontrivial dependence on interaction parameters is through the dependence of $\Lambda_{Q}^{s}$. As can be seen from Eqs. (36) and (37), this scaling function depends on the dimensionless groups $\kappa_{Q} D$ and $a / D$ only through the combination $\kappa_{Q}[a+D / 2]$. The scaling function $\Lambda_{Q}(X)$, with $X=\kappa_{Q}[a+D / 2]$ is plotted in Fig. 3(b). The scaling function can be represented to within $1 \%$ accuracy by the simple expression

$$
\Lambda^{s}(X)=\frac{22}{X^{2}+25 X+10},
$$

within the relevant range where $0.4 \leqslant X \leqslant 20$. This representation reduces the effort in the evaluation of the shadowing contribution to the diffusion coefficient considerably.

\section{WORKING EQUATIONS FOR DATA ANALYSIS: SUMMARY OF THE THEORY}

The following results from theory will be used to obtain the hydrodynamic screening length from experimental data for the long-time self-diffusion coefficient.

The concentration dependence of the diffusion coefficient $D_{s}$, relative to the Einstein diffusion coefficient $D_{0}$, is given by

$$
\frac{D_{s}}{D_{0}}=\frac{1}{1+\alpha_{\mathrm{isol}, \perp}^{h} \varphi} \frac{1}{1+\alpha_{\mathrm{iso}, \|, \perp}^{s} \varphi},
$$

where $\varphi$ is the hard-core volume fraction of rods, where the coefficients $\alpha_{\text {iso, } \|, \perp}^{h}$ describe the effect of screened hydrodynamic interactions and the coefficient $\alpha_{\text {iso, } \|, \perp}^{s}$ accounts for the shadowing effect; that is, the effect of direct interactions. The indices "iso," $\|$, and $\perp$ refer to diffusion in the isotropic and nematic states, parallel and perpendicular to the nematic director, respectively. In the shadowing contribution we wrote "by hand" the result in Eq. (28) in the form given in Eq. (39). The difference between the two expressions is small since $\alpha^{s} \varphi$ is small. The higher order terms present in the above expression might account in approximation for interactions with more than a single rod simultaneously. These higher order direct interactions need further investigation.

The hydrodynamic coefficients are equal to

$$
\alpha_{\text {iso }}^{h}=-\frac{\kappa a}{(\kappa D)^{2} \ln \{\kappa D\}}\left[\frac{64}{10}-\frac{85}{10000} \kappa L-\frac{33}{10000}(\kappa L)^{2}\right] \text {, }
$$

$$
\alpha_{\|}^{h}=-\frac{\kappa a}{(\kappa D)^{2} \ln \{\kappa D\}}\left[\frac{53}{10}-\frac{95}{1000} \kappa L+\frac{3}{10000}(\kappa L)^{2}\right] \text {, }
$$

$$
\alpha_{\perp}^{h}=-\frac{\kappa a}{(\kappa D)^{2} \ln \{\kappa D\}}\left[\frac{74}{10}-\frac{25}{1000} \kappa L-\frac{25}{10000}(\kappa L)^{2}\right] .
$$

These coefficients are functions of the dimensionless groups $\kappa a, \kappa D$, and $\kappa L$, where $\kappa^{-1}$ is the hydrodynamic screening length, $a$ is the radius of the tracer sphere, $D$ is the diameter of a rod, and $L$ is its length.

The shadowing coefficients are equal to

$$
\begin{aligned}
& \alpha_{\text {iso }}^{s}=R, \\
& \alpha_{\|}^{s}=R(1-S), \\
& \alpha_{\perp}^{s}=R\left(1+\frac{1}{2} S\right),
\end{aligned}
$$

where $S$ is the orientational order parameter (also denoted as $P_{2}$ ) and $R$ is given by 


$$
\begin{aligned}
R= & \frac{2}{3}\left(1+\frac{2 a}{D}\right)^{2}\left[1+2 \frac{l_{B}}{L} \frac{Z_{c} Z_{\mathrm{rod}}}{\left[1+\kappa_{Q} a\right]\left[1+\kappa_{Q} D / 2\right]}\right. \\
& \left.\times \frac{22}{\left(\kappa_{Q}[a+D / 2]\right)^{2}+25 \kappa_{Q}[a+D / 2]+10}\right],
\end{aligned}
$$

where $\kappa_{Q}^{-1}$ is the electrostatic Debye-Hückel screening length, $l_{B}$ is the Bjerrum length, and $Z_{c}$ and $Z_{\text {rod }}$ are the valencies of the colloidal tracer sphere and a rod, respectively. This coefficient is a function of the dimensionless groups $\kappa_{Q} a, \kappa_{Q} D, l_{B} / L, Z_{c}$, and $Z_{\text {rod. }}$.

The above expressions will be used in the following experimental part to obtain the hydrodynamic screening length from experimental data for the diffusion coefficient.

\section{EXPERIMENT}

In the following section the system of rigid rods (fdvirus particles) and the tracer sphere (apoferritin) is introduced. In Sec. V B, the ionic strength and $p \mathrm{H}$ of suspensions, and the surface charge of fd-virus particles and apoferritin are discussed. We always prepared our system through an osmotic equilibrium with a Tris/ $\mathrm{HCl}$ buffer of varying analytical concentration. The ionic strength of these osmotic reservoirs is the ionic strength from which the electrostatic Debye-Hückel screening length is calculated. The $p \mathrm{H}$ of these osmotic reservoirs is also the $p \mathrm{H}$ of the suspensions. This $p \mathrm{H}$ is affected by carbon dioxide that dissolves from the air when the analytical buffer concentration is smaller than about $10 \mathrm{mM}$. Section V B therefore contains a calculation of the $p \mathrm{H}$ as a function of the buffer concentration where dissolved carbon dioxide is taken into account. Since diffusion coefficients in the nematic state depend on the orientational order parameter, Sec. V C discusses data on the order parameter as a function of fd-particle concentration and ionic strength. Finally, in Sec. V D the diffusion data are presented. In the next section, all the results presented in the present section will be combined to extract the hydrodynamic screening length.

\section{A. The colloidal systems}

Bacteriophage $\mathrm{fd}$ is a rodlike molecule with a contour length of $L=880 \mathrm{~nm}$, a bare diameter of $D=6.6 \mathrm{~nm}$, a persistence length at high salt concentration of $P=2200 \mathrm{~nm}$, and a molecular weight of $M=1.64 \times 10^{7} \mathrm{~g} / \mathrm{mol}$. The charge density of fd-virus particles is discussed in detail in Sec. $\mathrm{V}$ B. The fd virus was grown and purified following standard biological protocols, ${ }^{32}$ using the XL1blue strain of E. coli as the host bacteria. The virus particles were purified by repeated centrifugation $\left(10^{5} \mathrm{~g}\right.$ for $\left.5 \mathrm{~h}\right)$ and finally redispersed in Tris/ $\mathrm{HCl}$ buffers with varying concentration, depending on the desired ionic strength.

Apoferritin is a protein with a diameter of $12.8 \mathrm{~nm}$, which is used here as a tracer sphere. The surface charge of apoferritin will be discussed in detail in Sec. V B.

The dimensions that are needed as an input in order to calculate the hydrodynamic screening length from diffusion data are

$$
D=6.6 \mathrm{~nm}, \quad L=880 \mathrm{~nm}, \quad a=6.4 \mathrm{~nm} .
$$

The volume fraction $\varphi$ of the fd virus can be related to the weight concentration $c$, using the linear dimensions in Eq. (45) and the molar mass $1.64 \times 10^{7} \mathrm{~g} / \mathrm{mol}$,

$$
\varphi=1.10 \times 10^{-3} c[\mathrm{mg} / \mathrm{ml}] .
$$

The numerical value of the weight concentration in this expression should be expressed in $\mathrm{mg} / \mathrm{ml}$, as indicated.

\section{B. Ionic strength, $\mathrm{pH}$, and surface charges}

As mentioned above, fd suspensions are dialyzed against a large reservoir of Tris/ $\mathrm{HCl}$ buffer with a known analytical concentration of Tris and added $\mathrm{HCl}$. The ionic strength that goes into the calculation of the Debye screening length is the ionic strength of the osmotic reservoir, being equal to the corresponding concentration in the dispersion outside the double layers. In order to calculate the Debye screening length, it is therefore sufficient to consider the ionic strength of the osmotic reservoir, without the presence of fd-virus particles. Furthermore, the activity of $\mathrm{H}^{+}$ions will be the same in the reservoir and the fd suspension, so that also the $p \mathrm{H}$ of the fd suspensions will be the same as in the reservoir, despite the fact that relatively many $\mathrm{H}^{+}$ions are dissociated from the surface of the fd-virus particles.

To prevent $\mathrm{CO}_{2}$ from dissolving in our samples would require working under a nitrogen atmosphere, which is not feasible for both the fluorescence correlation spectroscopy (FCS) and birefringence experiments. Exposure of low buffer-concentration samples for a few minutes to air was sufficient to affect their $p \mathrm{H}$. We therefore chose to equilibrate the suspensions and osmotic reservoirs with the surrounding air and to account for carbonic acid explicitly. For low ionic strengths (below Tris/ $\mathrm{HCl}$ buffer concentrations of $10 \mathrm{mM}$ ), the effect of dissolved $\mathrm{CO}_{2}$ on ionic strength and $\mathrm{pH}$ cannot be neglected. Both the $p \mathrm{H}$ and the ionic strength are important for the analysis of diffusion data in terms of the hydrodynamic screening length.

The calculation of ionic strength and $p \mathrm{H}$, where dissolved $\mathrm{CO}_{2}$ from the air is accounted for, is given in Appendix D. In this section we shall present the results of this calculation, together with experimental data which confirm this calculation.

The Tris $/ \mathrm{HCl}$ buffers are prepared by adding a small volume of $\mathrm{HCl}$ with a concentration of $1 M$ to a $20.0 \mathrm{mM}$ Tris solution till a $p \mathrm{H}$ of 8.2 is attained. This buffer is then diluted with de-ionized water for the preparation of the lower Tris/HCl-buffer concentrations. The analytical concentration $c_{T}$ of the Tris/ $\mathrm{HCl}$ buffer is defined as the total concentration of Tris:20 mM for the original buffer and $20 \mathrm{mM}$ divided by the dilution factor for the diluted buffers.

The $p \mathrm{H}$ is plotted in Fig. 4(a) against the analytical Tris/ $\mathrm{HCl}$ buffer concentration $c_{T}$. The solid line is the result of the calculations from Appendix D, including carbonic acid, which is in reasonable agreement with the measured $p \mathrm{H}$. The dashed line is the theoretical $p \mathrm{H}$ without taking $\mathrm{CO}_{2}$ into account. As can be seen, there is a significant influence of the $\mathrm{CO}_{2}$ that dissolves from the air for analytical buffer concentrations lower than $10 \mathrm{mM}$. As is shown in Fig. 4(b), where 

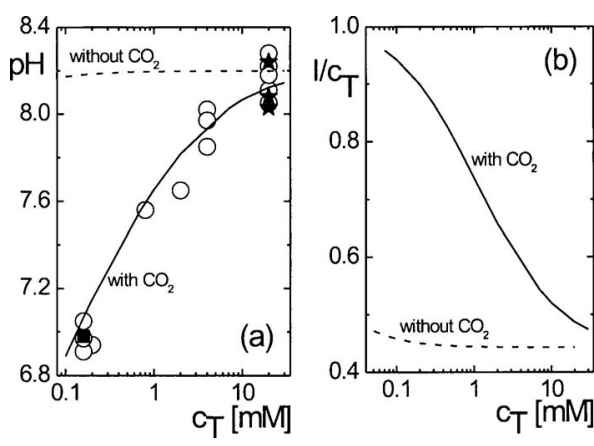

FIG. 4. (a) The $p \mathrm{H}$ as a function of the analytical Tris/ $\mathrm{HCl}$ buffer concentration $c_{T}$. The solid line is the theoretical prediction including carbonic acid from carbon dioxide that dissolves from the air, while the dashed line is the theoretical curve assuming that no $\mathrm{CO}_{2}$ dissolves in the buffer solutions. The data points $\bigcirc$ are experimental results. The $\boldsymbol{\square}$ is the $p \mathrm{H}$ for the $0.16 \mathrm{mM}$ dispersion with $3 \mathrm{mg} / \mathrm{ml} \mathrm{fd}$ virus, after dialysis. The $\star$ 's are the $p \mathrm{H}$ for the $20 \mathrm{mM}$ buffer solution with $100 \mathrm{mM} \mathrm{NaCl}$ added. The spread of the data points corresponds to the experimental errors both from the $p \mathrm{H}$ measurement itself and from the independent preparation of fresh buffer solutions. (b) The ratio $I / c_{T}$ of the ionic strength $I$ and the analytical buffer concentration $c_{T}$ as a function of $c_{T}$, as predicted with (solid line) and without (dashed line) dissolved $\mathrm{CO}_{2}$ from the air.

$I / c_{T}$ is plotted against $c_{T}$, the ionic strength is also significantly affected by $\mathrm{CO}_{2}$ for these lower buffer concentrations (the dashed line is the prediction without $\mathrm{CO}_{2}$ ). This is important for the calculation of the Debye screening length for the various Tris/ $\mathrm{HCl}$ buffers.

The data point $\square$ in Fig. 4(a) is the $p \mathrm{H}$ for the $0.16 \mathrm{mM}$ dispersion with the $3 \mathrm{mg} / \mathrm{ml}$ fd virus after dialysis. The majority of $\mathrm{H}^{+}$ions in the dispersion is due to the ions that are dissociated from the fd-virus particles and are for the larger fraction contained within the double layers. The $p \mathrm{H}$ of the dispersion, however, is the same as for the osmotic reservoir, as it should. The $\star$ in Fig. 4(a) is the $p \mathrm{H}$ for the $20 \mathrm{mM}$ buffer solution with $100 \mathrm{mM} \mathrm{NaCl}$ added. As can be seen, adding salt does not affect the $p \mathrm{H}$ within experimental accuracy.

Zimmermann et al. ${ }^{33}$ determined the charge of an fd virus, where titration curves are interpreted on the basis of different models for an fd virus particle. The model, where it is assumed that only the hydrophilic outer region of adsorbed proteins and not the DNA strand of fd itself is in contact with solvent, explains experimental titration curves almost perfectly (except for $p \mathrm{H}<4$ where the fd-virus particles are positively charged). From the data in Ref. 33 (see Fig. 5) it is found that in the $p \mathrm{H}$ range $7.0 \leqslant p \mathrm{H} \leqslant 8.2$ of our experiments, the charge of an fd-virus particle varies in the range $-10000 \leqslant Q_{\mathrm{fd}} \leqslant-8500$. Although the buffer capacity of the low ionic strength Tris/ $\mathrm{HCl}$ buffer against dissolving $\mathrm{CO}_{2}$ from the air is not sufficient to keep the $p \mathrm{H}$ at a value of 8.2, the $p \mathrm{H}$ range is still within a range where the surface charge of the fd-virus particles does not vary too much. The inset in Fig. 5 shows the charge dependence on $p \mathrm{H}$ of $\mathrm{fd}$ over a larger range.

Apoferritin has 624 negative and 576 positive dissociable groups. The isoelectric point is at $p \mathrm{H}=4$. Gapinski et $a l^{34}$ analyzed experimental structural and dynamical data on apoferritin using the charge as an adjustable parameter. They found that around $p \mathrm{H}=7$, the charge at very low apo-

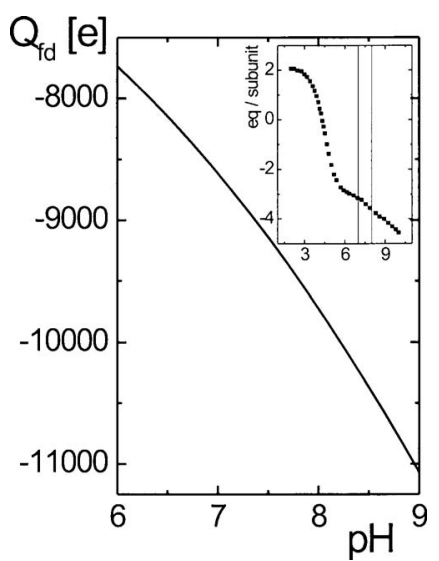

FIG. 5. The surface charge $Q_{\mathrm{fd}}$ of an fd-virus particle in units of the elementary charge $e>0$ as a function of the $p H$. Data are taken from Ref. 33. The inset shows the charge dependence (in units of eq/subunit) over a larger $p \mathrm{H}$ range. The vertical lines indicate the $p \mathrm{H}$ range relevant for the present study.

ferritin concentration varies from -5 to -10 elementary charges, depending on whether the charge is obtained from static or dynamic data. In Ref. 35 it is found from dynamic data that the charge is -3 for low apoferritin concentrations, increasing to about -10 at higher concentrations. Through the measurement of the second virial coefficient, Petsev and co-workers ${ }^{36-38}$ found a charge of -24 elementary charges at a $p \mathrm{H}$ of about 5. Since the charge of apoferritin is not unambiguously known, we shall use the charge in Sec. VI as a fit parameter such that the hydrodynamic screening length for isotropic networks for the various buffer concentrations collapses onto a single master curve. As it turns out, the charge determined in this way is -3 elementary charges.

\section{Orientational order parameters}

The setup with which the orientational order parameter has been determined is sketched in Fig. 6. The fd-virus suspension is aligned by means of a $2 \mathrm{~T}$ magnet with the magnetic field in the direction as indicated in Fig. 6. The magnetic field determines the orientation of the director and renders a monodomain nematic throughout the sample volume. A magnetic field of $2 \mathrm{~T}$ is too weak to affect the order parameter ${ }^{39,40}$ to within experimental error. The sample container is a quartz cuvette with a path length of $1 \mathrm{~mm}$. Before

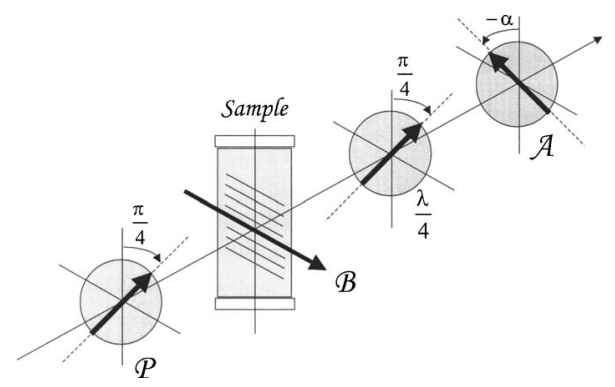

FIG. 6. The experimental setup for the determination of the orientational order parameter. The nematic sample is made monodomain by means of a magnetic field $B$. The incident polarized light is at $45^{\circ}$ with the vertical direction (polarizer $P$ ). After the sample a $\lambda / 4$ plate is oriented with one of its optical axis parallel with $P$. The transmitted intensity through the analyzer $A$ is minimized as a function of the angle $\alpha$. 
a measurement, the suspension is centrifuged for $1 \mathrm{~min}$ at $1000 \mathrm{~g}$ to remove possibly present dust. The incident laser beam is linearly polarized with an angle of $45^{\circ}$ with respect to the direction of the magnetic field (polarizer $P$ in Fig. 6). After the sample, there is a $\lambda / 4$ plate with one of its fast optical axis parallel to $P$. The transmitted intensity $I$ depends on the angle $\alpha$ of analyzer $A$ as

$$
I=\frac{1}{2} I_{0}[1+\sin \{2 \alpha+\Delta \Phi\}]
$$

where $I_{0}$ is the incident intensity and $\Delta \Phi$ is the retardation induced by the sample. Minimizing the intensity as a function of $\alpha$ by rotating the analyzer $A$ thus determines the retardation which, in turn, is equal to

$$
\Delta \Phi=2 \pi \frac{d}{\lambda} \Delta n,
$$

with $d=1 \mathrm{~mm}$ as the sample path length, $\lambda=633 \mathrm{~nm}$ the wavelength of the laser beam in vacuum, and $\Delta n$ the difference in refractive index along the principal axis of alignment of the fd-virus suspension. The orientational order parameter is equal to

$$
S=\Delta n / \Delta n_{\max },
$$

where $\Delta n_{\max }$ is the difference in refractive index if the fd rods were perfectly aligned. Clearly, $\Delta n_{\max }$ is proportional to the number concentration of rods. The saturation birefringence has been determined from magnetic birefringence measurements in Ref. 41 to be equal to 6.00 $\times 10^{-5} \mathrm{c} \mathrm{mg} / \mathrm{ml}$. From scattering data, a different value was found in Ref. 23,

$$
\Delta n_{\max }=[3.8 \pm 0.3] \times 10^{-5} c[\mathrm{mg} / \mathrm{ml}] .
$$

We shall use this latter expression for $\Delta n_{\max }$ since the earlier reported value in Ref. 41 turned out to give unphysical values for the order parameter. Equations (48)-(50) are used to calculate $S$ once $\Delta \Phi$ is found by minimizing the intensity in Eq. (47) with respect to $\alpha$.

The advantage of using a $\lambda / 4$ plate is that one can find $\Delta \Phi$ simply by minimizing the transmitted intensity. Without the $\lambda / 4$ plate, the transmitted intensity is equal to $\frac{1}{2} I_{0}[1$ $+\sin \{2 \alpha\} \cos \{\Delta \Phi\}]$. The determination of the retardation $\Delta \Phi$ in the absence of a $\lambda / 4$ plate thus requires a measurement of the incident intensity $I_{0}$. The use of a $\lambda / 4$ plate significantly increases the accuracy of the experimentally determined orientational order parameters.

The orientational order parameter $S$ is given in Fig. 7 as a function of the fd-virus concentration for the various analytical Tris/HCl-buffer concentrations and the sample with added $\mathrm{NaCl}$. For the lower Tris/ $\mathrm{HCl}$ concentrations, a magnetic field of $2 \mathrm{~T}$ was not able to induce a nematic monodomain above a certain fd concentration, indicated by " $\bigcirc$." For these higher fd concentrations at low ionic strengths, no measurements with the above described method could be performed. Below the concentration indicated by the vertical bar, the measured order parameter dropped sharply to a much lower value. This concentration is identified with the nematic-binodal concentration. The numerical values of the binodal concentrations obtained in this way are in accordance with those reported in Refs. 23, 40, and 42. Figure 7(f)
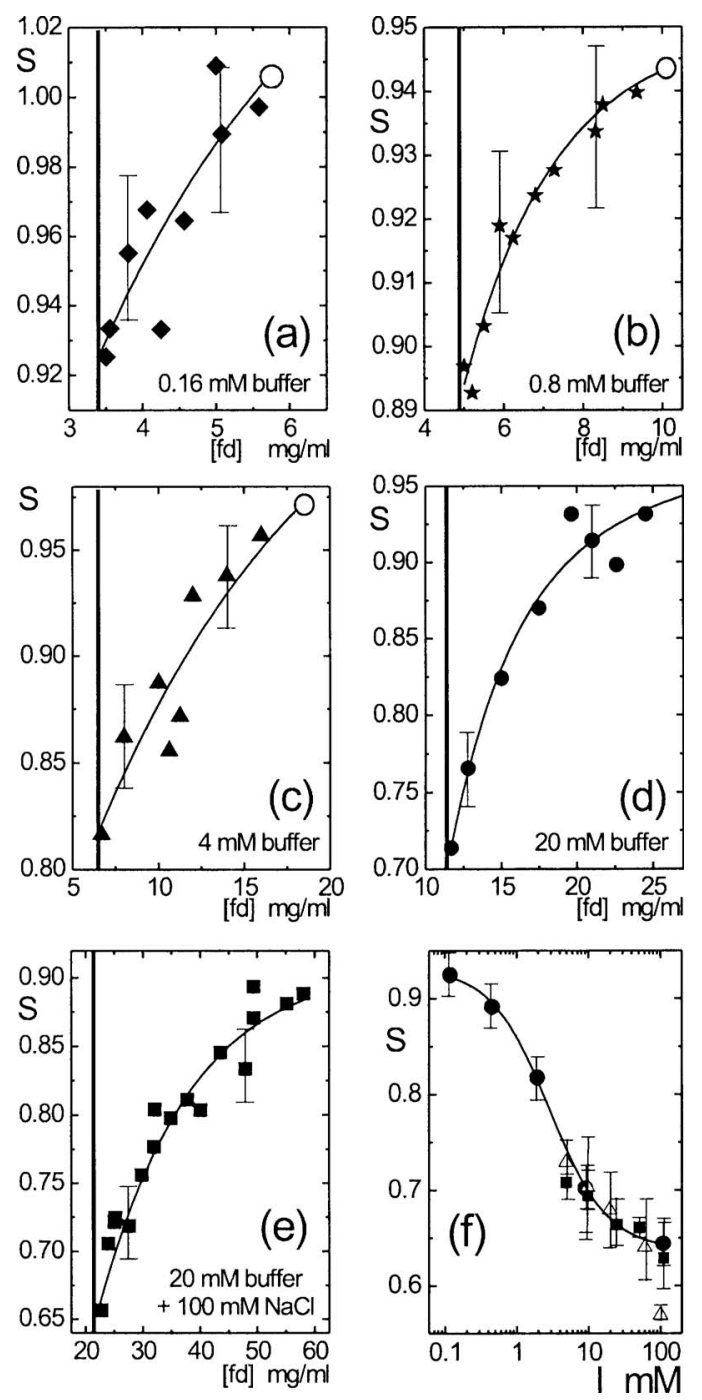

FIG. 7. The orientational order parameter $S$ as a function of fd-virus concentration for several Tris/HCl-buffer concentrations (and added salt), as indicated in the figures. The points $\bigcirc$ for the three lower salt concentrations indicate the concentration above which no monodomain could be obtained with the $2 \mathrm{~T}$ magnet. The solid curves are guides to the eye. The thick vertical line marks the upper binodal concentration. The lower right figure gives the value of the order parameter at the upper-binodal concentration as a function of ionic strength: $\operatorname{are}$ our data, $\triangle$ and $\mathbf{a r e}$ data from Refs. 23 and 42 , as obtained by birefringence and scattering measurements, respectively.

gives the order parameter at the nematic-binodal concentration as a function of ionic strength. As can be seen from this figure, our order parameters at the binodal concentration (the 's) agree with those reported earlier in Refs. 23 and 42.

The order parameter at the lowest salt concentration and the higher fd concentrations in Fig. 7(a) seems to asymptote to a value that is a little above unity. This is due either to about $1 \%-2 \%$ error in concentration or to the error in the value for the saturation birefringence in Eq. (50).

Within the Onsager theory, the order parameter, as well as the binodal concentrations, scale as $(L / D) \varphi \sim D L^{2}$. Scaling for various salt concentrations could therefore be achieved by introducing an effective concentration as $\nu_{\text {eff }}$ $\equiv[(L / D) \varphi]_{\mathrm{eff}}=\left(D_{\mathrm{eff}} / D\right) \nu$, where $\nu$ is the bare value of $[(L / D) \varphi]$, and the effective diameter $D_{\text {eff }}$ is equal to 


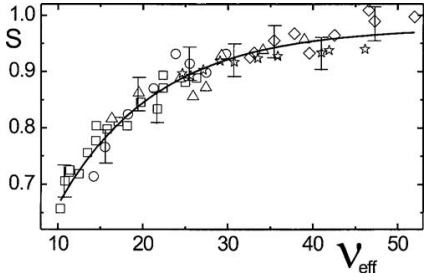

FIG. 8. Scaling of the order parameter with respect to an effective rod concentration $\nu_{\text {eff }}=[(L / D) \varphi]_{\text {eff }}$ with $\chi=15$, as described in the text. The open symbols correspond to the same filled symbols in Fig. 7 for the various buffer concentrations: $c_{T}=0.16 \mathrm{mM}(\diamond), 0.80 \mathrm{mM}(\succsim), 4.00 \mathrm{mM}(\triangle)$, $20.0 \mathrm{mM}(\bigcirc)$, and $20 \mathrm{mM}+100 \mathrm{mM} \mathrm{NaCl}(\square)$.

$$
D_{\text {eff }}=D\left[1+\frac{\chi}{\kappa_{Q} D}\right]
$$

Here, the number $\chi$ can be varied to achieve scaling. However, the fd-virus suspensions do not behave as Onsager systems. It is not possible to find a single value for $\chi$ such that the order parameters fall onto a master curve and at the same time have coinciding scaled binodal concentrations. Indeed, the order parameter at the binodal concentrations are different for the different ionic strengths [see Fig. 7(f)]. Deviations from the Onsager theory have also been observed in terms of the relative width of the biphasic region. ${ }^{20}$ Nevertheless, it turns out that our data for the order parameter can be scaled to within experimental error, as can be seen in Fig. 8, with $\chi=15$ in Eq. (51). The effective concentration $\nu_{\text {eff }}$ is thus related to the bare $\mathrm{fd}$ concentration in $\mathrm{mg} / \mathrm{ml}$ as

$$
\nu_{\text {eff }}=\left[1+\frac{15}{\kappa_{Q} D}\right] \nu=\left[1+\frac{15}{\kappa_{Q} D}\right] 0.147[f d],
$$

where we used Eqs. (45) and (46). To within experimental error, the data can be fitted with a single exponential function (see the solid line in Fig. 8). It is thus found that the fdconcentration dependence, as well as the salt-concentration dependence of the order parameter, can be described by the single scaling relation

$$
S=0.98-0.75 \exp \left\{-\left[1+\frac{15}{\kappa_{Q} D}\right] \times \frac{[f d]}{80}\right\},
$$

where the fd concentration should be expressed in $\mathrm{mg} / \mathrm{ml}$. This experimental result is used in the data analysis in Sec. VI.

It is not clear whether this scaling has any theoretical relevance. In the present paper, we shall use this scaling relation in our data analysis as a mere convenience in order to be able to calculate order parameters from the ionic strength and fd concentration. A discussion of the possible theoretical basis for the scaling relation (52) is beyond the scope of the present paper. A scaling of this sort has been discussed in Ref. 40. Note that the effective value of $(L / D) \varphi$ at the nematic-binodal concentration, for the highest ionic strength, is equal to 9.5, which is about a factor of 2 higher than the Onsager prediction of 4.2 in the case of stiff rods. Also, the corresponding order parameter at the binodal concentration is 0.65 as compared to 0.80 for stiff Onsager rods. The flexibility of the fd-virus particles is probably the reason that our experimental data for the highest ionic strength do not agree with those for stiff Onsager rods. Indeed, the experimental nematic-binodal concentration and order parameter at that concentration found here are in semiquantitative accordance with theoretical predictions for flexible hard-core rods with a chain flexibility of $L / P=0.4$, where $P$ $=2200 \mathrm{~nm}$ is the persistence length. ${ }^{43}$

The Debye screening length for water at $25^{\circ} \mathrm{C}$ is conveniently calculated from Eq. (20) as

$$
\kappa_{Q}^{-1}[\mathrm{~nm}]=0.304 / \sqrt{I[M]} .
$$

For our experiments, $\kappa_{Q} D$ varies from 0.24 , to 0.46 , to 0.96 , to 2.06 , and to 7.17 with increasing ionic strength, so that the factor $\chi / \kappa_{Q} D$ in Eq. (51) varies from 62, to 33 , to 16 , to 7.3 , and to 2.1 , respectively.

\section{Diffusion coefficients}

Tracer-diffusion constants of apoferritin in fd suspensions were measured with FCS. Since fd-virus particles fluoresce to some extent, the resulting contribution to the correlation function must be subtracted. How this is done is discussed in Ref. 15. Also, the sample container and monodomain alignment of the nematic samples is discussed in that reference. That FCS measures long-time self-diffusion coefficients for small tracer spheres has been shown in Ref. 14. It is also shown in Ref. 15 that the long-time selfdiffusion coefficient is equal to Fick's diffusion coefficient for mass transport, provided that the network structure is not affected by the presence of the small tracer spheres.

In Fig. 9 the measured diffusion coefficients are plotted as a function of $\mathrm{fd}$ concentration for the various analytical Tris/HCl-buffer concentrations that are used $\left(c_{T}=0.16,0.80\right.$, 4.00 , and $20.0 \mathrm{mM}$ and $c_{T}=20.0 \mathrm{mM}$ plus $100 \mathrm{mM} \mathrm{NaCl}$ ). The data for the highest ionic strength, where $100 \mathrm{mM} \mathrm{NaCl}$ is added, are taken from Ref. 15. The gray vertical regions indicate the location of the isotropic-nematic coexistence region. The same data, except for the highest ionic strength, are plotted in Fig. 10 as functions of fd concentration in the isotropic phase and in the nematic phase for diffusion perpendicular and parallel to the nematic director. As can be seen, there is a strong effect of the ionic strength on the diffusive behavior of apoferritin.

Note that the diffusion coefficients just below the isotropic binodal is in between the coefficients for parallel and perpendicular motion in the nematic state just above the isotropic binodal. As far as the shadowing effect is concerned, this is predicted by our theory [see Sec. IV, Eq. (43) in particular]. This difference between the self-diffusion coefficients for parallel and perpendicular motions is more pronounced for lyotropic rods (see Ref. 44), but of the same order as compared to thermotropic rods (see Ref. 45 and references therein).

\section{THE HYDRODYNAMIC SCREENING LENGTH}

The way to extract hydrodynamic screening lengths from the diffusion data is as follows. For a given fd concentration and salt concentration, the diffusion coefficient is calculated from the expressions in Sec. IV. The hydrodynamic screening length is chosen such that the calculated diffusion 

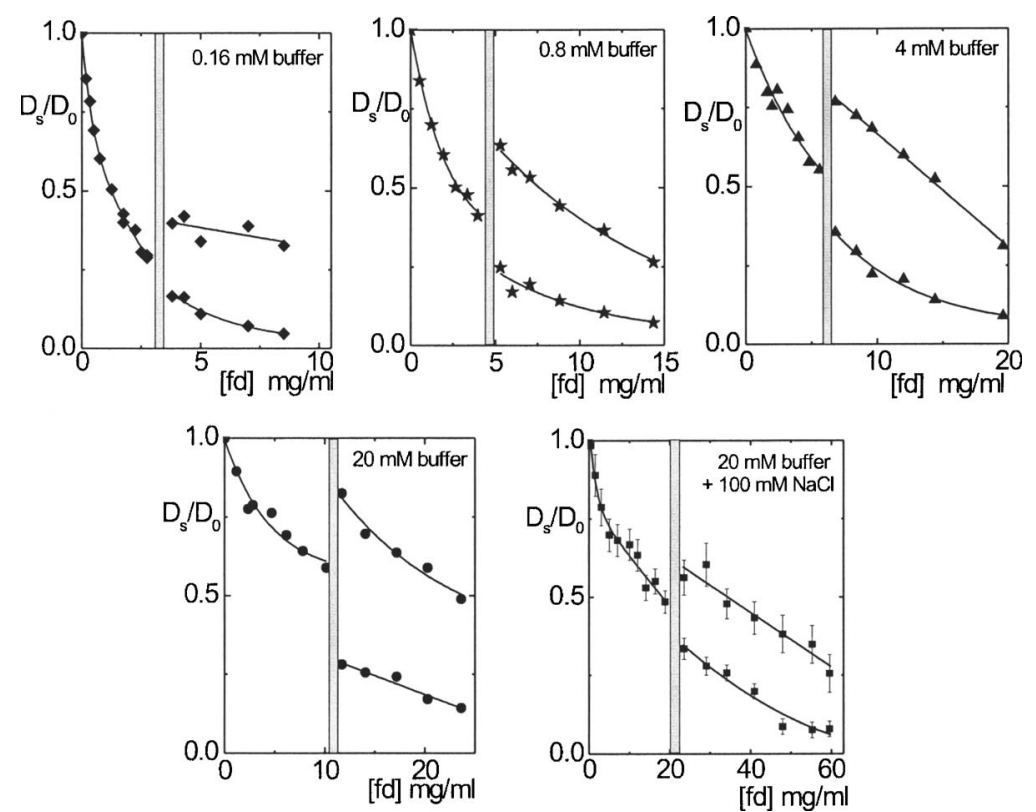

FIG. 9. Long-time self-diffusion coefficients of apoferritin in fd-virus particle suspensions as a function of fd concentration for various analytical Tris-buffer concentrations, as indicated. The last figure is for a $20.0 \mathrm{mM}$ Tris/HCl buffer with $100 \mathrm{mM}$ added $\mathrm{NaCl}$. These latter data are taken from Ref. 15. The vertical gray regions indicate the location of the IN biphasic region. In the nematic phase, the upper and lower curves relate to diffusions parallel and perpendicular to the director, respectively. The spread of data points around the solid curves which are drawn for a guide to the eye indicates the experimental errors. coefficient matches the experimentally measured value (the data in Figs. 9 and 10). The $p \mathrm{H}$ and ionic strength needed for the calculation of the diffusion coefficient are obtained for each analytical Tris/HCl-buffer concentration, as discussed in Sec. V B (see Fig. 4). The Debye screening length is then calculated according to Eq. (54), and the charge of the fdvirus particles is obtained from Fig. 5. In the case of nematic $\mathrm{fd}$ suspensions, the orientational order parameter for a given fd concentration and ionic strength is calculated from Eq. (53).

There are a few limitations on the validity of the theory developed above that exclude the use of some of the diffusion data for the calculation of the hydrodynamic screening length. Our analysis of the shadowing effect is limited to the case when the tracer sphere interacts with just one single rod, not with two or more rods simultaneously. This is only correct when the mesh size of the fd network is about ten times larger than the Debye screening length $\kappa_{Q}^{-1}$. Hence, we can only use diffusion data for which $\kappa_{Q} \xi \geqslant 10$. The mesh size can be calculated from

$$
\xi=L \sqrt{c^{*} / c},
$$

where $c^{*}=0.076 \mathrm{mg} / \mathrm{ml}$ is the fd-overlap concentration. For the same reason, our expression (28) for the shadowing ef-
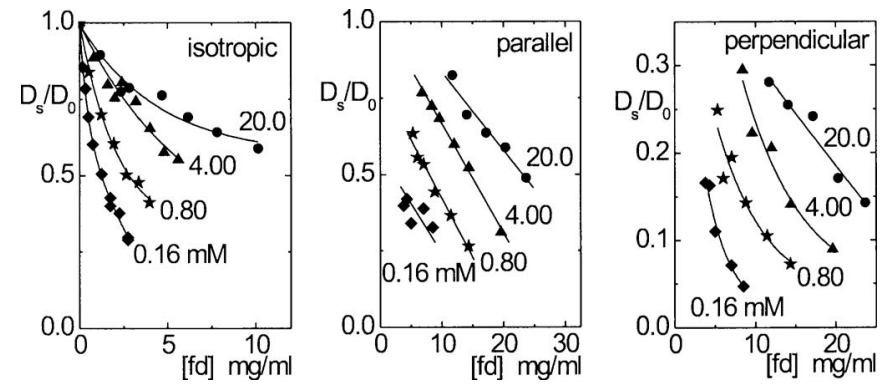

FIG. 10. Diffusion coefficients of apoferritin in fd-virus particle suspensions as a function of fd concentration in the isotropic state and the nematic state, for diffusions perpendicular and parallel to the nematic director, for various analytical Tris-buffer concentrations. The data with the added $\mathrm{NaCl}$ (last figure in Fig. 9) are not included for clarity. fect is the first order term in an expansion with respect to the fd concentration. The combination $\alpha_{\text {iso, }, \perp \|}^{s} \varphi$ should therefore be less than about $1 / 2$. A third requirement is that the hydrodynamic screening length is at least about two times larger than the mesh size; that is, $\kappa \xi \leqslant 1 / 2$. Data points that do not meet these requirements should not be included in the data analysis where our theory is applied.

As mentioned in Sec. V B, the surface charge $Z_{c}$ of apoferritin is not precisely known. We therefore use $Z_{c}$ as a fitting parameter, such that the data for the isotropic phase for all buffer concentrations collapse onto a single master curve. The best choice for $Z_{c}$ turns out to be $Z_{c}=-3$. This charge agrees with that reported in Ref. 35 for low apoferritin concentrations and is lower than the values reported in Refs. 34 and $36-38$, where charges varying from -5 to -24 have been found. The resulting master curve is given in Fig. 11(a), where the screening length is plotted in units of the rod length versus the fd concentration, and in Fig. 11(b), where the screening length is given in units of the mesh size of the network as a function of the mesh size. For the high buffer concentrations, the above-mentioned requirements for the validity of the theory are satisfied for most data points, while the hydrodynamic screening length is insensitive to the

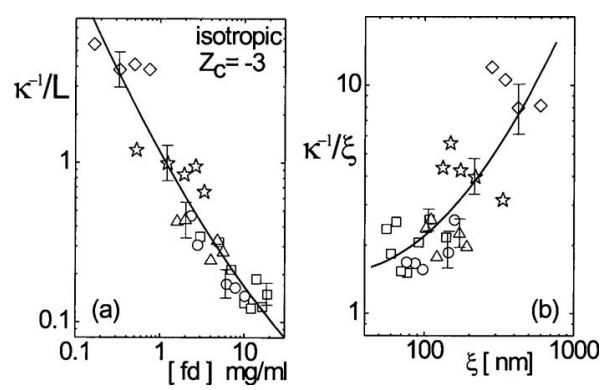

FIG. 11. (a) The hydrodynamic screening length $\kappa^{-1}$ in units of the length $L$ of the rods vs the fd concentration in the isotropic phase. (b) The screening length in units of the mesh size as a function of the mesh size. Here, $Z_{c}=$ -3 and the symbols refer to the different buffer concentrations: $c_{T}$ $=0.16 \mathrm{mM}(\diamond), 0.80 \mathrm{mM}($ 污) $, 4.00 \mathrm{mM}(\triangle), 20.0 \mathrm{mM}(\bigcirc)$, and $20 \mathrm{mM}$ $+100 \mathrm{mM} \mathrm{NaCl}(\square)$. The solid curves are guides to the eye. 

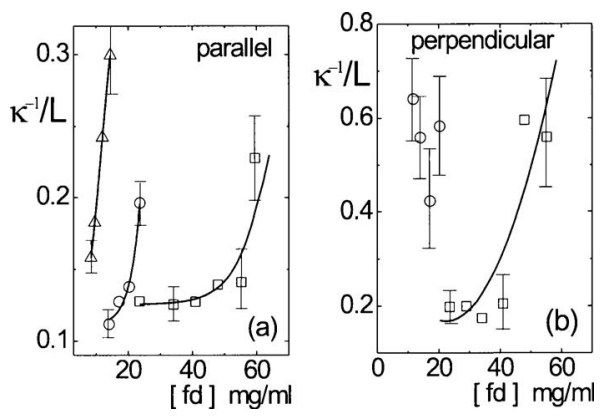

FIG. 12. The hydrodynamic screening length $\kappa^{-1}$ in units of the length $L$ of the rods vs the fd concentration for the nematic phase parallel (a) and perpendicular (b) to the director. The symbols refer to the different buffer concentrations: $c_{T}=4.00 \mathrm{mM}(\triangle), 20.0 \mathrm{mM}(\bigcirc)$, and $20 \mathrm{mM}+100 \mathrm{mM}$ $\mathrm{NaCl}(\square)$. Error bars correspond to the experimental errors in the measured diffusion coefficients.

precise value for the charge of apoferritin. However, for the two lower buffer concentrations (the points $\diamond$ and $i$ in Fig. 11 ), the Debye screening length becomes too large as compared to the mesh size for the higher fd concentrations (the points where $\kappa_{Q} \xi<10$ are not included in Fig. 11). This is why the data points for the two lower buffer concentrations in Fig. 11 tend to deviate a bit from the master curve on increasing the fd concentration. Note that the hydrodynamic screening length increases with increasing mesh size considerably faster than the mesh size itself [see Fig. 11(b)].

The hydrodynamic screening lengths for the nematic phase are given in Figs. 12(a) and 12(b) for diffusion parallel and perpendicular to the director, respectively. The reason for the absence of data for lower ionic strengths is that the above-mentioned requirements are not met. The experimental errors for the $20 \mathrm{mM}$ buffer concentration and perpendicular diffusion are too large to draw a line to guide the eye. Two conclusions can be drawn from Fig. 12. First of all, the hydrodynamic screening length for the perpendicular case is larger than that for the parallel case. This implies that shear waves created by a tracer sphere that moves perpendicular to the director penetrates the nematic network deeper as compared to a sphere moving parallel to the director. Secondly, hydrodynamic screening lengths considerably increase at a given fd concentration when the ionic strength is decreased. This is due to the increase of orientational order of the $\mathrm{fd}$ suspensions on decreasing the ionic strength. For the same reason, the screening length increases for a given ionic strength on increasing the fd concentration. In the nematic state, the decrease of the screening length due to increasing fd concentration is overruled by the much greater increase of the screening length due to the increasing orientational order. As can be seen in Figs. 12(a) and 7, the screening length for the parallel case increases by a factor of 3 (from $\kappa^{-1} / L$ $=0.1$ to about 0.3 ) at an fd concentration of $14 \mathrm{mg} / \mathrm{ml}$ on increasing the order parameter from 0.71 to 0.90 . For the perpendicular case, the screening length increases by a factor of 3 to 4 (from $\kappa^{-1} / L=0.15$ to about 0.55 ) at an fd concentration of $23 \mathrm{mg} / \mathrm{ml}$ on increasing the order parameter from 0.65 to about 0.8 . There is thus a very sensitive dependence of the hydrodynamic screening length on the orientational order parameter.
Since the rods are very much longer than the (anisotropic) mesh size of the nematic network, the rods are probably very much entangled also in the nematic state. Hydrodynamic interactions are therefore also screened in the nematic networks. There is, however, no direct evidence for strong entanglement in these nematic fd networks. Since we are probing tracer diffusion of a sphere that is much smaller than the mesh size of the network, the different screening lengths of the rod network that are found in directions parallel and perpendicular to the nematic director cannot be attributed to the different viscosities in these two directions.

\section{SUMMARY AND CONCLUSION}

A theory is developed for tracer diffusion of a sphere through networks of long and thin stiff rods, with the inclusion of interactions between the tracer sphere and the network due to charges. The tracer sphere is assumed to be small as compared to the mesh size of the network. Screened hydrodynamic interactions, hard-core interactions, and charge interactions between the tracer sphere and the rod network are accounted for. Simple scaling relations are derived, where the effects of hydrodynamic interactions and direct interactions decouple in the sense that the diffusion coefficient is a product of two factors which are entirely determined by hydrodynamics and direct interactions. Longtime diffusion coefficients have been measured by means of fluorescence correlation spectroscopy for various ionic strengths as a function of the rod concentration. As a tracer sphere, we used apoferritin and the rod network consists of fd-virus particles. In the nematic phase, just above the binodal, the diffusion coefficients for motion of the tracer sphere parallel and perpendicular to the director are typically a factor of 2 different. Order parameters have been measured for the same ionic strengths, again as a function of the rod concentration. These data extend earlier measurements to lower ionic strengths. At these lower ionic strengths (below about $1 \mathrm{mM}$ ), the order parameter at the nematic-binodal concentration can be as large as 0.9. A simple empirical scaling relation is found. It describes both the ionic-strength and rod-concentration dependences of the orientational order parameter. The hydrodynamic screening length is extracted from the experimental diffusion coefficients and order parameters, using the scaling relations that account for hydrodynamic as well as direct interactions. For the isotropic networks, the hydrodynamic screening lengths for all ionic strengths as a function of rod concentration collapse on a single curve. Here, the charge of the apoferritin particle is taken to be equal to -3 elementary charges, which is of the same order as found in independent studies. In the nematic networks, the screening length is found to increase with increasing rod concentration for the ionic strengths for which the theory can be applied. This is due to a pronounced increase of the screening length on increasing the orientational order parameter, as is clear from a comparison of the screening length for different ionic strengths.

As yet, there is no independent theory for the hydrodynamic screening length in the isotropic of nematic networks of rods. In particular, the master curve for the screening 
length as a function of the rod concentration for isotropic networks cannot yet be obtained from an independent theory. Also, the origin of the sensitive dependence of the screening length on the orientational order parameter of nematic networks is not yet understood.

The range of validity of the present theory can in principle be extended to relatively larger Debye lengths as compared to the mesh size by considering the shadowing effect where the sphere is allowed to instantaneously interact with more than just a single rod. Such an extended theory would allow us to extract the hydrodynamic screening length from our data at lower ionic strengths as well. This remains a future challenge.

Erratum to Ref. 15. An error is made in Ref. 15 in the calculation of $L_{\mathrm{hc}}$. In transforming the Smoluchowski equation [Eq. (14)] to cylindrical coordinates, the middle term on the left-hand side in Eq. (16) was erroneously omitted. All results in Ref. 15 remain correct when $\nu$ there is set equal to 1 (instead of the erroneous value of $1.618 \cdots$ ). Furthermore, for the high salt case considered in Ref. 15, the shadowing effect is very small, so that the quantitative results in that reference are not affected by this error.

\section{ACKNOWLEDGMENTS}

This work is done within the framework of the Transregio-SFB "Colloids in External Fields" and the European Network of Excellence SoftComp.

\section{APPENDIX A: SOLUTION OF EQUATION (16)}

The function $L(\rho)$ is written as $L_{\mathrm{hc}}(\rho)+L_{Q}(\rho)$, where the hard-core contribution $L_{\mathrm{hc}}$ is given by Eq. (17) and $L_{Q}$ is the additional contribution due to charge interactions. Since $g_{0}$ $=g_{0, \mathrm{hc}} g_{0, Q}$, where $g_{0, \mathrm{hc}}=\exp \left\{-\beta V_{\mathrm{hc}}\right\}$ is the hard-core paircorrelation function, and $g_{0, Q}=\exp \left\{-\beta V_{Q}\right\}$, Eq. (16) can be written as

$$
\begin{aligned}
& g_{0, \mathrm{hc}}(\rho) {\left[\frac{d L_{Q}^{2}(\rho)}{d \rho^{2}}+\frac{1}{\rho} \frac{d L_{Q}(\rho)}{d \rho}-\frac{L_{Q}(\rho)}{\rho^{2}}\right] } \\
&=\left\{\beta g_{0, \mathrm{hc}}(\rho) \frac{d V_{Q}(\rho)}{d \rho}-\frac{d g_{0, \mathrm{hc}}(\rho)}{d \rho}\right\} \\
& \times\left[\beta+\frac{d L_{\mathrm{hc}}(\rho)}{d \rho}+\frac{d L_{Q}(\rho)}{d \rho}\right] .
\end{aligned}
$$

This differential equation can be cast into an integral equation as follows. First note that for $\rho>a+D / 2$ this reduces to

$$
\begin{aligned}
& \frac{d L_{Q}^{2}(\rho)}{d \rho^{2}}+\frac{1}{\rho} \frac{d L_{Q}(\rho)}{d \rho}-\frac{L_{Q}(\rho)}{\rho^{2}} \\
& =\beta \frac{d V_{Q}(\rho)}{d \rho}\left[\beta+\frac{d L_{\mathrm{hc}}(\rho)}{d \rho}+\frac{d L_{Q}(\rho)}{d \rho}\right] .
\end{aligned}
$$

The substitution of $L_{Q}(\rho)=C(\rho) L_{\mathrm{hc}}(\rho)$ as an ansatz into Eq. (A2) and the use of $d L_{\mathrm{hc}}(\rho) / d \rho=-L_{\mathrm{hc}}(\rho) / \rho$ give

$$
\frac{d}{d \rho}\left[L_{\mathrm{hc}}(\rho) \frac{d C(\rho)}{d \rho}\right]=\beta \frac{d V_{Q}(\rho)}{d \rho}\left[\beta-\frac{L_{\mathrm{hc}}(\rho)}{\rho}+\frac{d L_{Q}(\rho)}{d \rho}\right] .
$$

Integration thus leads to

$$
\begin{aligned}
C(\rho)= & A+\int_{a+D / 2}^{\rho} d \rho^{\prime} L_{\mathrm{hc}}^{-1}\left(\rho^{\prime}\right) \\
& \times\left\{B+\beta \int_{a+D / 2}^{\rho^{\prime}} d \rho^{\prime \prime} \frac{d V_{Q}\left(\rho^{\prime \prime}\right)}{d \rho^{\prime \prime}}\right. \\
& \left.\times\left[\beta-\frac{L_{\mathrm{hc}}\left(\rho^{\prime \prime}\right)}{\rho^{\prime \prime}}+\frac{d L_{Q}\left(\rho^{\prime \prime}\right)}{d \rho^{\prime \prime}}\right]\right\},
\end{aligned}
$$

where $A$ and $B$ are integration constants. Since $L_{Q}(\rho) \rightarrow 0$ for $\rho \rightarrow \infty$, the term within the curly brackets should vanish as $\rho^{\prime} \rightarrow \infty$. This sets $B$ equal to

$$
B=-\beta \int_{a+D / 2}^{\infty} d \rho^{\prime \prime} \frac{d V_{Q}\left(\rho^{\prime \prime}\right)}{d \rho^{\prime \prime}}\left[\beta-\frac{L_{\mathrm{hc}}\left(\rho^{\prime \prime}\right)}{\rho^{\prime \prime}}+\frac{d L_{Q}\left(\rho^{\prime \prime}\right)}{d \rho^{\prime \prime}}\right],
$$

and, hence,

$$
\begin{aligned}
C(\rho)= & A-\beta \int_{a+D / 2}^{\rho} d \rho^{\prime} L_{\mathrm{hc}}^{-1}\left(\rho^{\prime}\right) \int_{\rho^{\prime}}^{\infty} d \rho^{\prime \prime} \frac{d V_{Q}\left(\rho^{\prime \prime}\right)}{d \rho^{\prime \prime}} \\
& \times\left[\beta-\frac{L_{\mathrm{hc}}\left(\rho^{\prime \prime}\right)}{\rho^{\prime \prime}}+\frac{d L_{Q}\left(\rho^{\prime \prime}\right)}{d \rho^{\prime \prime}}\right] .
\end{aligned}
$$

The integration constant $A$ can be determined similarly as for the hard-core case considered in Ref. 15. The integration of Eq. (A1) from $a+D / 2-\epsilon$ to $a+D / 2+\epsilon$ with vanishing $\epsilon$, using $d g_{\mathrm{hc}}(\rho) / d \rho=\delta(\rho-a-D / 2) \quad$ (with $\delta$ the onedimensional delta distribution), gives

$$
\frac{d L(\rho=a+D / 2)}{d \rho}=-\beta .
$$

Since $d L_{\mathrm{hc}}(\rho=a+D / 2) / d \rho=-\beta$, it follows that $d L_{Q}(\rho=a$ $+D / 2) / d \rho=0$. Equation (A6) and $L_{Q}=C L_{\mathrm{hc}}$ shows that $A$ $=B / \beta$, where $B$ is given in Eq. (A5). The resulting integral equation for $L_{Q}$ is most conveniently written as

$$
L_{Q}(\rho)=-L_{\mathrm{hc}}(\rho)\left[F^{c}+\beta \int_{a+D / 2}^{\rho} d \rho^{\prime} L_{\mathrm{hc}}^{-1}\left(\rho^{\prime}\right) F\left(\rho^{\prime}\right)\right],
$$

where

$$
F\left(\rho^{\prime}\right)=\int_{\rho^{\prime}}^{\infty} d \rho^{\prime \prime} \frac{d V_{Q}\left(\rho^{\prime \prime}\right)}{d \rho^{\prime \prime}}\left[\beta-\frac{L_{\mathrm{hc}}\left(\rho^{\prime \prime}\right)}{\rho^{\prime \prime}}+\frac{d L_{Q}\left(\rho^{\prime \prime}\right)}{d \rho^{\prime \prime}}\right]
$$

and $F^{c}=F\left(\rho^{\prime}=a+D / 2\right)$ is the contact value of $F\left(\rho^{\prime}\right)$. Disregarding the second order term $\sim\left(d V_{Q} / d \rho\right)\left(d L_{Q} / d \rho\right)$ in the integral in Eq. (A9) finally leads to expression (22) for $L_{Q}(\rho)$.

\section{APPENDIX B: DERIVATION OF EQUATION (24)}

Using the probability density function for the position coordinate of the tracer sphere is equal to $g / V$ (with $V$ as the volume of the system), transforming to cylindrical coordi- 
nates as defined in Fig. 2, and the substitution of representation (15) for the pair-correlation function readily leads to

$$
\begin{aligned}
\langle\nabla V\rangle=-\pi \bar{\rho} L \mathbf{F}^{\operatorname{ext}} \int_{0}^{\infty} d \rho \rho g_{0}(\rho) L(\rho) \frac{d V(\rho)}{d \rho}, \\
\langle\nabla \ln \{g\}\rangle=-\pi \bar{\rho} L \mathbf{F}^{\operatorname{ext}} \int_{0}^{\infty} d \rho \rho \\
\times\left[g_{0}\left\{\frac{d L(\rho)}{d \rho}+\frac{L(\rho)}{\rho}\right\}+L(\rho) \frac{d g_{0}(\rho)}{d \rho}\right] .
\end{aligned}
$$

The transformation to cylindrical coordinates as defined in Fig. 2 proceeds in a similar way as described in Appendix B of Ref. 15.

To evaluate the ensemble averages, first note that $g_{0}$ $=g_{0, \mathrm{hc}} g_{0, Q}$, with $g_{0, \mathrm{hc}}=\exp \left\{-\beta V_{\mathrm{hc}}\right\}$ as the hard-core part of the pair-correlation function (with $V_{\mathrm{hc}}$ as the hard-core interaction potential) and $g_{0, Q}=1-\beta V_{Q}$ as the contribution due to the charge interactions to leading order. Useful relations for the calculation of the ensemble averages are

$$
\begin{aligned}
& g_{0, \mathrm{hc}}(\rho)=H(\rho-a-D / 2), \\
& \exp \left\{-\beta V_{\mathrm{hc}}(\rho)\right\} d V_{\mathrm{hc}}(\rho) / d \rho=-\beta^{-1} \delta(\rho-a-D / 2), \\
& d L_{\mathrm{hc}}(\rho) / d \rho=-L_{\mathrm{hc}}(\rho) / \rho
\end{aligned}
$$

where $H$ is the Heaviside function and $\delta$ is the delta distribution. From these relations it is easily verified that to first order in charge-interaction contributions,

$$
\begin{aligned}
\langle\nabla V\rangle= & -\pi \bar{\rho} L \mathbf{F}^{\operatorname{ext}}\left\{-\beta^{-1}(a+D / 2)\left(L_{\mathrm{hc}}^{c}+L_{Q}^{c}-\beta L_{\mathrm{hc}}^{c} V_{Q}^{c}\right)\right. \\
& \left.+\int_{a+D / 2}^{\infty} d \rho \rho \frac{d V_{Q}(\rho)}{d \rho} L_{\mathrm{hc}}(\rho)\right\}
\end{aligned}
$$

where, as before, the contact value $H^{c}$ of a function $H$ of $\rho$ is defined as $H^{c}=H(\rho=a+D / 2)$. Using form (17) for $L_{\mathrm{hc}}$ in the integral, this expression reduces to

$$
\langle\nabla V\rangle=\pi \bar{\rho} L \mathbf{F}^{\mathrm{ext}} \beta^{-1}(a+D / 2)\left(L_{\mathrm{hc}}^{c}+L_{Q}^{c}\right) .
$$

The substitution of Eqs. (22) and (23) finally leads to Eq. (24) for $\langle\nabla V\rangle$.

Next, consider the ensemble average of $\nabla \ln g$. The substitution of $g_{0}=\exp \left\{-\beta V_{\mathrm{hc}}\right\} g_{0, Q}$ into Eq. (B1) and the use of relations (B2) give

$$
\begin{aligned}
\langle\nabla \ln g\rangle= & \int_{a+D / 2}^{\infty} d \rho \rho\left[g_{0, Q}(\rho)\left\{\frac{d L(\rho)}{d \rho}+\frac{L(\rho)}{\rho}\right\}\right. \\
& \left.+L(\rho) \frac{d g_{0, Q}(\rho)}{d \rho}\right]+\left(a+\frac{D}{2}\right) L^{c} g_{0, Q}^{c} .
\end{aligned}
$$

Since

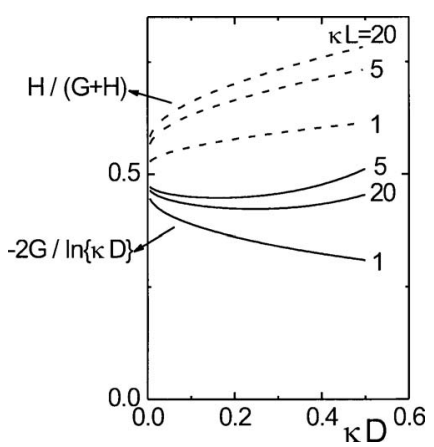

FIG. 13. The combinations $G / 1 / 2 \ln \{\kappa D\}$ (solid curves) and $H /(G+H)$ (dotted curves) vs $\kappa D$ for various values of $\kappa L$.

$$
\begin{aligned}
\int_{a+D / 2}^{\infty} & d \rho \rho L(\rho) \frac{d g_{0, Q}(\rho)}{d \rho} \\
= & -\left(a+\frac{D}{2}\right) L^{c} g_{0, Q}^{c}-\int_{a+D / 2}^{\infty} d \rho g_{0, Q}(\rho) \frac{d}{d \rho}[\rho L(\rho)],
\end{aligned}
$$

it follows that $\langle\nabla \ln g\rangle=0$.

\section{APPENDIX C: THE DEPENDENCE OF $\Lambda^{h}$ ON $\kappa D$ AND $S$}

The scaling functions $\Lambda_{\mathrm{iso}, \|, \perp}^{h}$ are independent of $\kappa D$ for those values of $\kappa D$ and $\kappa L$ where the functions $G$ and $H$ in Eq. (6) are such that $G$ is equal to $\frac{1}{2} \ln \{\kappa D\}$ and $H /(G+H)$ is equal to $1 / 2$. The functions $G / \frac{1}{2} \ln \{\kappa D\}$ and $H /(G+H)$ are plotted in Fig. 13 versus $\kappa D$ for various values of $\kappa L$. As can be seen, these functions reach their limiting values to within about $5 \%$ for $\kappa D<0.05$ for all $\kappa L$ in the range of $1-20$. Within the range of validity of the Debye-Büche-Brinkman approach, we can therefore use the limiting forms of the two above-mentioned functions.

Next, consider the explicit orientational-order-parameter dependence of $\alpha^{h}$ that enters through the orientational pdf in Eq. (9). Numerical results for $\alpha^{h}$ are given in Fig. 14, for the nematic networks parallel and perpendicular to the nematic director, for the parameters pertaining to fd virus and apoferritin. These numerical results are obtained using the following Gaussian form for the orientational pdf (Ref. 45):
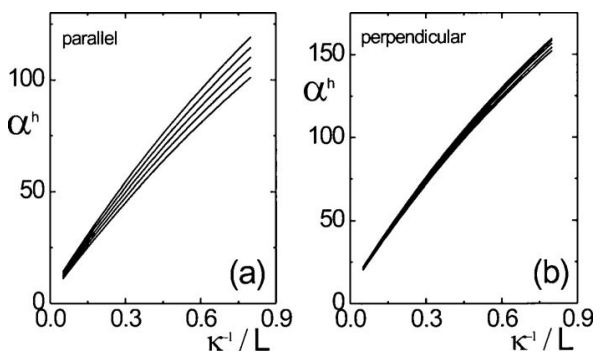

FIG. 14. (a) The coefficient $\alpha_{\|}^{h}$ (a) and $\alpha_{\perp}^{h}$ (b) for the parameters pertaining to fd virus and apoferritin, as a function of $\kappa^{-1} / L$ for various orientational order parameters $0.5,0.6,0.7,0.8$, and 0.9 from top to bottom in (a) and bottom to top in (b). 


$$
\begin{aligned}
& P_{0}(\widetilde{\mathbf{u}} \mid S)=\frac{1}{N(C)}\left[\exp \left\{-C \Theta^{2}\right\}+\exp \left\{-C(\pi-\Theta)^{2}\right\}\right], \\
& 0 \leqslant \Theta<\pi,
\end{aligned}
$$

where $\Theta$ is the angle between $\hat{\mathbf{u}}$ and the nematic director. The parameter $C$ measures the width of the orientational distribution and is set by the scalar orientational order parameter $S$. Furthermore, $N$ is the normalization constant, which is also a function of $S$. This representation has also been used in Ref. 15 for similar experiments at high salt concentrations.

As can be seen, $\alpha^{h}$ is only weakly dependent on the orientational order parameter once the hydrodynamic screening length is fixed. That is, the orientational-order-parameter dependence of $\alpha^{h}$ is essentially only due to the implicit orientational-order-parameter dependence of the hydrodynamic screening length $\kappa^{-1}$. To a good approximation, $\alpha^{h}$ is therefore only a function of $\kappa^{-1} / L$, which shows that $\Lambda^{h}$ is independent of $S$ as far as the explicit dependence through the orientational pdf is concerned. This weak explicit dependence on the orientational order parameter has also been verified for other parameters than those for fd virus and apoferritin.

\section{APPENDIX D: THE EFFECT OF $\mathrm{CO}_{2}$ ON IONIC STRENGTH AND $p H$}

Let $\mathrm{H}_{2} \mathrm{CO}_{3}$ denote dissolved, hydrated carbon dioxide. The concentration of hydrated $\mathrm{CO}_{2}$ is directly proportional to the partial pressure $p_{\mathrm{CO}_{2}}$ of gaseous $\mathrm{CO}_{2}$ in the air,

$$
\left[\mathrm{H}_{2} \mathrm{CO}_{3}\right]=K_{\mathrm{CO}_{2}} p_{\mathrm{CO}_{2}},
$$

where $[X]$ denotes the concentration of a substance $X$ in $M$ $\equiv$ moles $/ \mathrm{dm}^{3}$. The constant $K_{\mathrm{CO}_{2}}$ is independent of the $p \mathrm{H}$ since it describes the mere solvation of gaseous carbon dioxide. Carbonic acid will dissociate in $\mathrm{HCO}_{3}^{-}$and $\mathrm{CO}_{3}^{2-}$. The mass-action laws for these dissociation reactions are

$$
\begin{aligned}
& \frac{\left[\mathrm{H}^{+}\right]\left[\mathrm{HCO}_{3}^{-}\right]}{\left[\mathrm{H}_{2} \mathrm{CO}_{3}\right]}=K_{\mathrm{C}_{1}}=\left(4.3 \times 10^{-7}\right) M, \\
& \frac{\left[\mathrm{H}^{+}\right]\left[\mathrm{CO}_{3}^{2-}\right]}{\left[\mathrm{HCO}_{3}^{-}\right]}=K_{\mathrm{C}_{2}}=\left(5.6 \times 10^{-11}\right) M,
\end{aligned}
$$

where the acid constants $K_{\mathrm{C}_{j}}$ are independent of the $p \mathrm{H}$ and ionic strength.

Unprotonated and protonated Tris will be denoted as $T$ and $T^{+}$, respectively. The mass-action law for the Trisprotonation reaction is

$$
\frac{\left[\mathrm{H}^{+}\right][T]}{\left[T^{+}\right]} \equiv K_{T}=\left(7.9 \times 10^{-9}\right) M .
$$

Again, the acid constant $K_{T}$ is independent of the $p \mathrm{H}$ and ionic strength.

Neglecting the very small concentration of undissociated $\mathrm{HCl}$, the remaining relations that are necessary to calculate the $p \mathrm{H}$ and ionic strength are

$$
\begin{aligned}
& 2\left[\mathrm{CO}_{3}^{2-}\right]+\left[\mathrm{HCO}_{3}^{-}\right]+\left[\mathrm{Cl}^{-}\right]+\left[\mathrm{OH}^{-}\right]=\left[T^{+}\right]+\left[\mathrm{H}^{+}\right], \\
& {\left[\mathrm{Cl}^{-}\right]=c_{a},} \\
& {[T]+\left[T^{+}\right]=c_{T},} \\
& {\left[\mathrm{H}^{+}\right]\left[\mathrm{OH}^{-}\right] \equiv K_{w}=10^{-14.0} M^{2},}
\end{aligned}
$$

where $c_{T}$ and $c_{a}$ denote the total (or "analytical") concentration of Tris and $\mathrm{HCl}$ in units of $M \equiv$ moles $/ \mathrm{dm}^{3}$, respectively (the subscripts stand for Tris and acid). The first equation expresses electroneutrality, the second and third equations are conservation equations, while the fourth equation is the mass-action law for the dissociation of water.

From the above equations it is readily found that the $\mathrm{H}^{+}$ concentration is the solution of the following quartic equation:

$$
\begin{gathered}
{\left[\mathrm{H}^{+}\right]^{4}+\left[\mathrm{H}^{+}\right]^{3}\left(c_{T}-c_{a}+K_{T}\right)-\left[\mathrm{H}^{+}\right]^{2}\left(K_{w}+c_{a} K_{T}\right)-\left[\mathrm{H}^{+}\right] K_{w} K_{T}} \\
=K_{\mathrm{C}_{1}} K_{\mathrm{CO}_{2}} p_{\mathrm{CO}_{2}}\left\{\left[\mathrm{H}^{+}\right]^{2}+\left[\mathrm{H}^{+}\right]\left(2 K_{\mathrm{C}_{2}}+K_{T}\right)+2 K_{\mathrm{C}_{2}} K_{T}\right\} .
\end{gathered}
$$

The ionic strength is equal to

$$
I=\frac{1}{2}\left\{\left[\mathrm{H}^{+}\right]+\left[\mathrm{OH}^{-}\right]+\left[T^{+}\right]+\left[\mathrm{Cl}^{-}\right]+\left[\mathrm{HCO}_{3}^{-}\right]+4\left[\mathrm{CO}_{3}^{2-}\right]\right\},
$$

where the various concentrations can be expressed in terms of $\left[\mathrm{H}^{+}\right]$from Eqs. (D1)-(D4) as

$$
\begin{aligned}
& {\left[\mathrm{OH}^{-}\right]=K_{w} /\left[\mathrm{H}^{+}\right],} \\
& {\left[T^{+}\right]=c_{T}\left[\mathrm{H}^{+}\right] /\left(\left[\mathrm{H}^{+}\right]+K_{T}\right),} \\
& {\left[\mathrm{Cl}^{-}\right]=c_{a},} \\
& {\left[\mathrm{HCO}_{3}^{-}\right]=K_{\mathrm{C}_{1}} K_{\mathrm{CO}_{2}} p_{\mathrm{CO}_{2}} /\left[\mathrm{H}^{+}\right],} \\
& {\left[\mathrm{CO}_{3}^{2-}\right]=K_{\mathrm{C}_{1}} K_{\mathrm{C}_{2}} K_{\mathrm{CO}_{2}} p_{\mathrm{CO}_{2}} /\left[\mathrm{H}^{+}\right]^{2} .}
\end{aligned}
$$

The ionic strength can thus be calculated once $\left[\mathrm{H}^{+}\right]$is obtained as the (numerical) solution of Eq. (D5).

The value of the constant $K_{\mathrm{CO}_{2}} p_{\mathrm{CO}_{2}}$ can be used as a fitting parameter to the experimental $p \mathrm{H}$ data. A value of $0.015 \mathrm{mM}$ gives rise to the solid curves in Fig. 4. Tabulated values for the solubility of gaseous $\mathrm{CO}_{2}$ refer to the total amount of dissolved $\mathrm{CO}_{2}$, including $\mathrm{HCO}_{3}^{-}$and $\mathrm{CO}_{3}^{2-}$. That is, Henry's constant $H_{\mathrm{CO}_{2}}$, defined as

$$
\left[\mathrm{H}_{2} \mathrm{CO}_{3}\right]+\left[\mathrm{HCO}_{3}^{-}\right]+\left[\mathrm{CO}_{3}^{2-}\right]=H_{\mathrm{CO}_{2}} p_{\mathrm{CO}_{2}},
$$

is tabulated for pure air. The $p \mathrm{H}$ dependence of Henry's constant $H_{\mathrm{CO}_{2}}$ can be obtained from the mass-action laws [Eq. (D2)]. Eliminating $\left[\mathrm{HCO}_{3}^{-}\right]$and $\left[\mathrm{CO}_{3}^{2-}\right]$ in Eq. (D8) in favor of $\left[\mathrm{H}^{+}\right]$and $\left[\mathrm{H}_{2} \mathrm{CO}_{3}\right]$ gives

$$
\left[\mathrm{H}_{2} \mathrm{CO}_{3}\right]\left\{1+\frac{K_{\mathrm{C}_{1}}}{\left[\mathrm{H}^{+}\right]}+\frac{K_{\mathrm{C}_{1}} K_{\mathrm{C}_{2}}}{\left[\mathrm{H}^{+}\right]^{2}}\right\}=H_{\mathrm{CO}_{2}} p_{\mathrm{CO}_{2}} .
$$

Comparing to Eq. (D1) thus leads to 


$$
K_{\mathrm{CO}_{2}}=H_{\mathrm{CO}_{2}} /\left\{1+\frac{K_{\mathrm{C}_{1}}}{\left[\mathrm{H}^{+}\right]}+\frac{K_{\mathrm{C}_{1}} K_{\mathrm{C}_{2}}}{\left[\mathrm{H}^{+}\right]^{2}}\right\} .
$$

This equation specifies the $p \mathrm{H}$ dependence of Henry's constant $H_{\mathrm{CO}_{2}}$. Tabulated values for Henry's constant do not often specify the $p \mathrm{H}$ at which they are measured. The value that we find for $K_{\mathrm{CO}_{2}}$, however, is of the same order as one would find from tabulated values of $\mathrm{H}_{\mathrm{CO}_{2}}$ together with Eq. (D10) using reasonable values for the $p \mathrm{H}$.

The Tris/ $\mathrm{HCl}$ buffers are prepared by adding a small volume of $\mathrm{HCl}$ with a concentration of $1 M$, to a $20.0 \mathrm{mM}$ Tris solution till a $p \mathrm{H}$ of 8.2 is attained. This buffer is then diluted with de-ionized water for the preparation of the lower Tris/ $\mathrm{HCl}$ buffer concentrations. The analytical concentration $c_{a}$ of $\mathrm{HCl}$ after attaining a $p \mathrm{H}$ of 8.2 of the $20.0 \mathrm{mM}$ Tris solution is found from the above equations to be equal to

$$
c_{a}=8.85 \mathrm{mM} \text { for the } 20.0 \mathrm{mM} \text { Tris/ } \mathrm{HCl} \text { buffer. }
$$

The analytical concentrations $c_{a}$ and $c_{T}$ for the diluted buffers are simply equal to the initial values $8.85 \mathrm{mM}$ for $c_{a}$ and $20.0 \mathrm{mM}$ for $c_{T}$, divided by the dilution factor.

${ }^{1}$ S. Gorti and B. R. Ware, J. Chem. Phys. 83, 6449 (1985).

${ }^{2}$ J. D. Jones and K. Luby-Phelps, Biophys. J. 71, 2742 (1996).

${ }^{3}$ C. F. Schmidt, M. Bärmann, G. Isenberg, and E. Sackmann, Macromolecules 22, 3638 (1989).

${ }^{4}$ O. Seksek, J. Biwersi, and A. S. Verkman, J. Cell Biol. 138, 131 (1997).

${ }^{5}$ Y. Shafrir, D. Ben-Avraham, and G. Forgacs, J. Cell. Sci. 113, 2747 (2000).

${ }^{6}$ M. Arrio-Dupont, G. Foucault, M. Vacher, P. F. Deveaux, and S. Cribier, Biophys. J. 78, 901 (2000).

${ }^{7}$ J. Apgar, Y. Tseng, E. Fedorov, M. B. Herwig, S. C. Almo, and D. Wirtz, Biophys. J. 79, 1095 (2000).

${ }^{8}$ Y. Tseng and D. Wirtz, Biophys. J. 81, 1643 (2001).

${ }^{9}$ A. S. Verkman, Trends Biochem. Sci. 27, 27 (2002).

${ }^{10}$ I. Y. Wong, M. L. Gardel, D. R. Reichman, E. R. Weeks, M. T. Valentine, A. R. Bausch, and D. A. Weitz, Phys. Rev. Lett. 92, 178101 (2004).

${ }^{11}$ S. Mangenot, S. Keller, and J. Rädler, Biophys. J. 85, 1817 (2003).

${ }^{12}$ J. van der Gucht, N. A. M. Besseling, W. Knoben, L. Bouteiller, and M. A. Cohen Stuart, Phys. Rev. E 67, 051106 (2003).

${ }^{13}$ G. H. Koenderink, S. Sacanna, D. G. A. Aarts, and A. P. Philipse, Phys. Rev. E 69, 021804 (2004).

${ }^{14}$ K. Kang, J. Gapinski, M. P. Lettinga, J. Buitenhuis, G. Meier, M. Ratajczyk, J. K. G. Dhont, and A. Patkowski, J. Chem. Phys. 122, 044905 (2005).

${ }^{15}$ K. Kang, A. Wilk, J. Buitenhuis, A. Patkowski, and J. K. G. Dhont, J.
Chem. Phys. 124, 044907 (2006).

${ }^{16}$ C. Graf, H. Kramer, M. Deggelmann, M. Hagenbüchle, C. Johner, C. Martin, and R. Weber, J. Chem. Phys. 98, 4920 (1993).

${ }^{17}$ F. G. Schmidt, B. Hinner, E. Sackmann, and J. X. Tang, Phys. Rev. E 62, 5509 (2000).

${ }^{18}$ T. A. J. Lenstra, Z. Dogic, and J. K. G. Dhont, J. Chem. Phys. 114, 10151 (2001).

${ }^{19}$ K.-H. Lin, J. C. Crocker, A. C. Zeri, and A. G. Yodh, Phys. Rev. Lett. 87, 088301 (2001).

${ }^{20}$ J. Tang and S. Fraden, Liq. Cryst. 19, 459 (1995).

${ }^{21}$ M. Adams, Z. Dogic, S. L. Keller, and S. Fraden, Nature (London) 393, 349 (1998).

${ }^{22}$ Z. Dogic and S. Fraden, Langmuir 16, 7820 (2000).

${ }^{23}$ K. R. Purdy, Z. Dogic, S. Fraden, A. Rühm, L. Lurio, and S. G. J. Mochrie, Phys. Rev. E 67, 031708 (2003)

${ }^{24}$ S. Fraden, in Observation, Prediction and Simulation of Phase Transitions in Complex Fluids, NATO Advanced Studies Institute, Series C: Mathematical and Physical Sciences Vol. 460, edited by M. Baus, L. F. Rull, and J. P. Ryckaert (Kluwer Academic, Dordrecht, 1995), p. 113.

${ }^{25}$ Z. Dogic and S. Fraden, in Soft Matter, Complex Colloidal Suspensions, edited by G. Gompper and M. Schick (Wiley-VCH, Weinheim, 2006), Vol. 2, p. 1 .

${ }^{26}$ Z. Dogic and S. Fraden, Curr. Opin. Colloid Interface Sci. 11, 47 (2006).

${ }^{27}$ R. I. Cukier, Macromolecules 17, 252 (1984).

${ }^{28}$ A. R. Altenberger, M. Tirrell, and J. S. Dahler, J. Chem. Phys. 84, 5122 (1986).

${ }^{29}$ A. G. Ogston, B. N. Preston, and J. D. Wells, Proc. R. Soc. London A333, 297 (1973).

${ }^{30}$ J. Han and J. Herzfeld, Biophys. J. 65, 1155 (1993).

${ }^{31}$ B. Bagchi and S. Bhattacharyya, Adv. Chem. Phys. 116, 67 (2001)

${ }^{32}$ J. Sambrook, E. F. Fritsch, and T. Maniatis, Molecular Cloning: A Laboratory Manual (Cold Spring Harbor Laboratory, New York, 1989).

${ }^{33}$ K. Zimmermann, J. Hagedorn, C. C. Heuck, M. Hinrichsen, and J. Ludwig, J. Biol. Chem. 261, 1653 (1986).

${ }^{34}$ J. Gapinski, A. Wilk, A. Patkowski, W. Häussler, A. J. Banchio, R. Pecora, and G. Nägele, J. Chem. Phys. 123, 054708 (2005).

${ }^{35}$ W. Häussler, A. Wilk, J. Gapinski, and A. Patkowski, J. Chem. Phys. 117, 413 (2002).

${ }^{36}$ D. N. Petsev and P. G. Vekilov, Phys. Rev. Lett. 84, 1339 (2000).

${ }^{37}$ D. N. Petsev, B. R. Thomas, S.-T. Yau, and P. G. Vekilov, Biophys. J. 78, 2060 (2000).

${ }^{38}$ V. N. Paunov, E. W. Kaler, S. I. Sandler, and D. N. Petsev, J. Colloid Interface Sci. 240, 640 (2001).

${ }^{39}$ J. Tang and S. Fraden, Phys. Rev. Lett. 71, 3509 (1993).

${ }^{40}$ J. Tang and S. Fraden, Liq. Cryst. 19, 459 (1995).

${ }^{41}$ J. Torbet and G. Maret, Biopolymers 20, 2657 (1981).

${ }^{42}$ K. R. Purdy and S. Fraden, Phys. Rev. E 70, 061703 (2004).

${ }^{43}$ Z. Y. Chen, Macromolecules 26, 3419 (1993).

${ }^{44}$ M. P. Lettinga, E. Barry, and Z. Dogic, Europhys. Lett. 71, 692 (2005).

${ }^{45}$ F. Noack, in Physical Properties of Liquid Crystals, edited by D. Demus, J. Goodby, G. W. Gray, H.-W. Spiess, and V. Vill (Wiley-VCH, Weinheim, 1999), p. 480. 